\title{
Synthèse bibliographique : micro-texturation et microinjection de thermoplastiques
}

Julie Vera ${ }^{1, *}$, Anne-Catherine Brulez ${ }^{1,2}$, Elise Contraires ${ }^{1}$, Mathieu Larochette ${ }^{1}$, Stéphane Valette ${ }^{1}$, and Stéphane Benayoun ${ }^{1}$

${ }^{1}$ Laboratoire de Tribologie et Dynamique des Systèmes, UMR CNRS 5513, École Centrale de Lyon, 36 avenue Guy-de-Collongues, 69134 Écully cedex, France

${ }^{2}$ Laboratoire de Génie de la Fonctionnalisation des Matériaux Polymères, Institut Textile et Chimique de Lyon, 87 chemin des Mouilles, 69134 Écully cedex, France

Reçu le 29 mai 2017 / Accepté le 3 octobre 2017

Résumé. La fonctionnalisation de surface des matériaux et notamment des polymères fait l'objet de recherches intenses dans de nombreux secteurs tels que l'industrie du biomédical ou du transport afin de conférer aux pièces des propriétés spécifiques comme l'antibuée, la réduction du frottement ou le dégivrage... Dans le cas d'une production en grande série de pièces polymères fonctionnalisées, il est préférable, pour des questions de coûts, de générer des textures, au moyen d'une technique de reproduction d'empreinte comme l'injection plastique. Toutefois les fonctions requises nécessitent parfois la reproduction de dimensions microniques voire submicroniques poussant à ses limites la maîtrise du procédé conventionnel, avec les caractéristiques de l'injection de micro-pièces, mais aussi des spécificités propres à la micro-texturation. L'objet de cette revue bibliographique est de couvrir le large spectre des problèmes techniques et scientifiques associés à la microtexturation des pièces plastiques. Les techniques d'usinage de ces micro-motifs sur les outillages et le rôle des revêtements est particulièrement décrit ainsi que le besoin de mettre en œuvre des approches spécifiques de caractérisation topographique des textures. L'influence des paramètres du procédé d'injection est aussi discutée, soulignant la nécessité d'appréhender la micro-texturation des pièces plastiques avec une nouvelle grille de lecture de la microinjection.

Mots clés : microinjection / réplication / revêtement / texturation / paramètres procédés

\begin{abstract}
Micro-texturing and microinjection of thermoplastics: a review. For several years, scientists try to confer specific properties to the surfaces of materials (anti-fogging, reduction of friction, defrosting, etc.). Thus, the surface functionalization of materials and specially polymer, is the subject of intensive research in many industries such as the biomedical industry or the transportation industry. In the case of large-scale production of functionalized polymer parts, it is preferable, for reasons of cost, to generate patterns using a replication technique. However, the required functions can require pattern reproductions with micronic or submicronic lengths which push to its limits the control of the conventional process, with micro-injection characteristics, but also specificities of micro-texturing process. The objectives of this review is to cover a broad range of technical and scientific problems associated to micro-texturing polymer parts. The machining technique of these micro-patterns on the mold and the coating role is particularly described as well as the need to carry out specific approaches of topographic characterization. The influence of the injection process parameters is also discussed in detail, emphasizing the necessity to have a new approach to control micro-texturing polymer parts by injection molding.
\end{abstract}

Keywords: microinjection molding / replication / coating / texturing / process parameters

\footnotetext{
* e-mail: julie.vera@hotmail.com
} 


\section{Introduction}

Le marché mondial de la plasturgie réalisait en 2014 un chiffre d'affaires de 851 milliards d'euros dont 29 milliard d'euros en France [1]. C'est un secteur très concurrentiel qui produit souvent des pièces en grande série. La prise de nouvelles parts de marché pour des industriels est souvent concomitante à la création d'innovation et au développement de produits à haute valeur ajoutée. Parmi ces enjeux technologiques, la maîtrise des dimensions submicroniques sur les pièces plastiques revêt un caractère tout particulier. Cela se décline tout autant dans le domaine des micro-technologies ou de la plastronique par la fabrication de micro-pièces [2], que dans des secteurs plus conventionnels où les enjeux sont de conférer aux pièces une diversité de fonctions qui nécessitent des texturations de surface aux motifs de plus en plus fins. Les approches biomimétiques depuis ces vingt dernières années ont, de ce point de vue, ouvert de nouveaux champs d'applications et aiguisées les imaginations [3]. Parmi ces applications on note les traitements hydrophobes inspirés de l'effet lotus [4-6], les texturations anti-buées [7-9], comme celles générant des effets optiques (modification de la couleur, codage d'informations...) que l'on retrouve naturellement sur les ailes d'un papillon le Morpho menelaus [10].

Généralement une micro-pièce en matière plastique est définie comme suit [11]:

- une pièce de quelques milligrammes;

- une pièce dont certaines de ses tolérances sont de l'ordre du micromètre;

- une pièce sur laquelle on réalise des détails de l'ordre du micromètre. Dans ce cas, si le détail est de l'ordre du nanomètre, on parlera de nano-injection même si la pièce est de taille conventionnelle.

Cette micro-pièce peut alors être réalisée soit directement (usinage de motifs micrométriques par enlèvement de matière...) soit de manière indirecte par réplication. Récemment des techniques d'extrusion [12] ont permis d'élaborer des multicouches dont les épaisseurs n'excèdent pas quelques centaines de nanomètres mais cela ne peut se généraliser à des motifs aux formes complexes [13].

On entend par procédé de réplication une méthode qui consiste à transférer sur la pièce la géométrie d'une empreinte établie sur un moule ou une matrice. Les techniques génériques de reproduction en masse de micro/ nano-structures sont principalement l'embossage et l'injection dont les principes sont schématisés (Tab. 1).

Les premières pièces thermoplastiques (PVC) micromoulées ont été réalisées par embossage en 1970 [14]. L'objectif de ce travail était de développer une nouvelle technique de reproduction de films en hologrammes pour la télévision. Ces travaux furent ensuite poursuivis par une équipe de Zurich [15] où des réseaux de diffraction pour le filtrage des couleurs ont été réalisés par embossage à chaud du PVC. Le rapport profondeur sur largeur des microstructures était au maximum de 5,7 (1,4 $\mu \mathrm{m}$ de hauteur pour $0,4 \mu \mathrm{m}$ de large). Dans le milieu des années 1980, la technologie LIGA qui était initialement l'acronyme de «Lithografie und Galvanik» qui signifie «lithographie et électrodéposition» fut initiée en Allemagne [16]. Elle permit la réalisation de textures à la surface du moule avec une résolution inférieure à $0,1 \mu \mathrm{m}$. Des travaux sur le moulage par injection réactive [17] puis sur le procédé d'injection de polymères [18] ont suivi.

Les procédés d'embossage peuvent être séquentiels avec le démoulage de chaque pièce mais ils peuvent être aussi continus, en texturant des bandes de polymères sous l'effet d'une compression exercée par des matrices cylindriques à l'image de celles rencontrées dans l'impression des journaux. Dans ce cas, il s'agit d'embosser la matière juste après l'extrusion [19].

Le principe du moulage par injection consiste à remplir très rapidement les cavités froides d'un moule avec de la matière plastique à l'état fondu. La matière plastique s'écoule dans le moule pour en épouser les formes. Les parois froides du moule permettent d'évacuer les calories, ce qui conduit à la solidification du polymère. La pièce ainsi mise en forme est démoulée après refroidissement et solidification de la matière plastique. Le tableau 2 présente quelques caractéristiques techniques comparatives des deux types de procédés pour la fabrication de nanostructures, l'embossage et l'injection.

Concernant les limites dimensionnelles des nanostructures atteintes par ces technologies, les résultats sont relativement proches. L'injection permet, en revanche, de réaliser des pièces aux formes plus complexes que l'embossage tel que des évidements ou des contredépouilles. En laboratoire, l'embossage à chaud peut être préféré car c'est une technique flexible et plus simple à mettre en œuvre. Le procédé d'injection est, quant à lui, le procédé le plus fréquemment utilisé en industrie car c'est une technique qui reste moins coûteuse dans un contexte de grande série [23] (voir Tab. 3).

Ainsi une approche plus globale de sélection des procédés, pour conférer de nouvelles fonctions à un ensemble très large de pièces plastiques et à grande échelle, montre toute la pertinence de s'attacher à la micro/nanotexturation des pièces par injection, c'est ce que nous développerons dans la suite de l'exposé.

En injection, une bonne reproduction des motifs dépend des caractéristiques du polymère, des caractéristiques du moule et des paramètres du procédé comme le schématise la figure 1. Ainsi après avoir présenté rapidement le procédé d'injection, Section 2, on s'attachera à expliquer en quoi les caractéristiques du polymère (Sect. 3) et de l'outillage (Sect. 4) influent sur la qualité finale de la pièce. Dans la Section 5 , nous exposerons comment les conditions de mise en œuvre du polymère impactent la qualité de réplique. Cet article fait le point sur l'état de l'art de la réplication submicroniques par injection. Nous nous sommes limités préférentiellement aux articles publiés ces vingt dernières années.

\section{Généralités sur le procédé d'injection}

Le moulage par injection est la méthode la plus courante de mise en œuvre des polymères et notamment les polymères thermoplastiques. Il représente en 2015, $53 \%$ de la production française de pièces plastique [1] et cette 
Tableau 1. Schémas de principe de reproduction de textures par thermoformage et injection.

Table 1. Schematic drawing of reproduction of textures by thermoforming and injection.

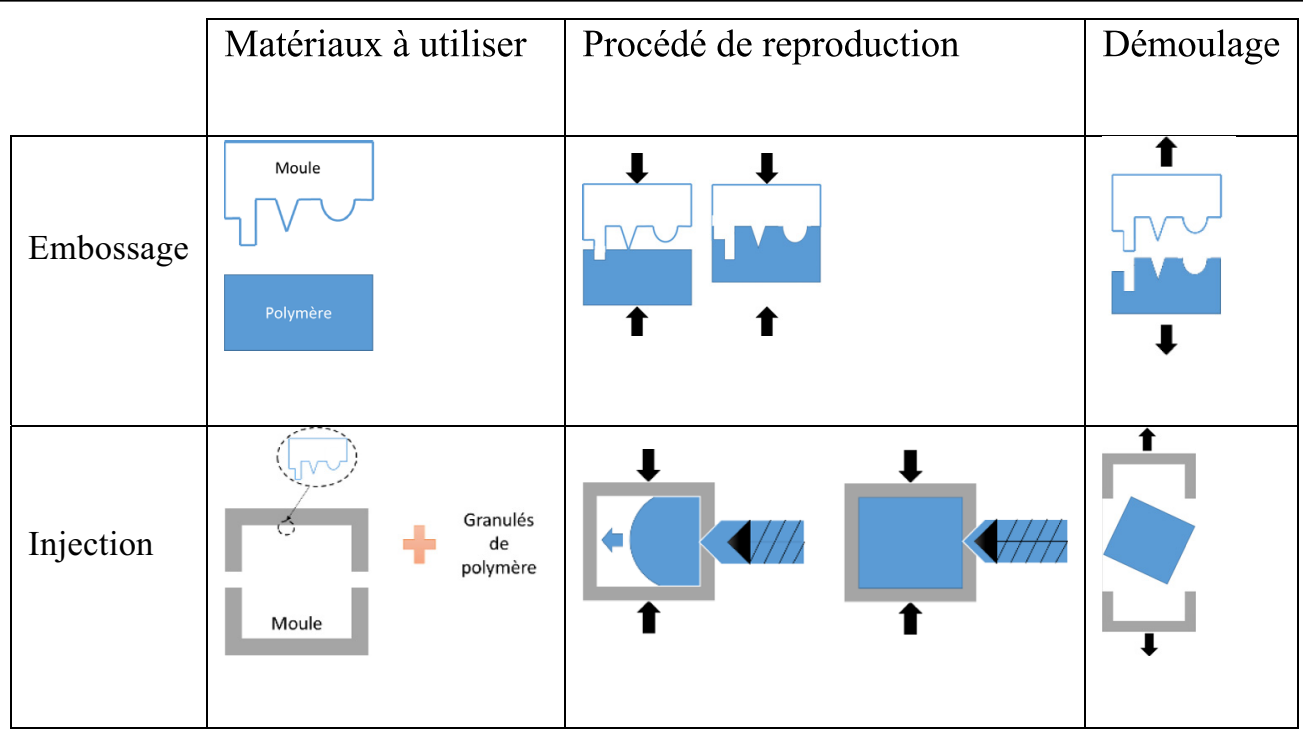

Tableau 2. Techniques de reproduction des nanostructures.

Table 2. Reproduction techniques of nanostructures.

\begin{tabular}{lllll}
\hline Procédés & $\begin{array}{l}\text { Dimensions minimale } \\
\text { des textures } \\
(\mu \mathrm{m})\end{array}$ & $\begin{array}{l}\text { Rapport hauteur/ } \\
\text { largeur des structures }\end{array}$ & $\begin{array}{l}\text { Polymères } \\
\text { (matériau de la pièce) }\end{array}$ & Références \\
\hline $\begin{array}{l}\text { Embossage à chaud } \\
\text { Injection }\end{array}$ & 0,2 & 10 & $\begin{array}{l}\text { PC, PET, PMMA } \\
\text { La grande majorité des } \\
\text { thermoplastiques polymères }\end{array}$ & $\begin{array}{l}{[20-22]} \\
\text { voir tableau } 5\end{array}$ \\
\hline
\end{tabular}

Tableau 3. Indice de prix en fonction des quantités produites, (ce sont des ordres de grandeur en fonction d'un indice de prix unitaire), d'après [24].

Table 3. Price index according to the quantities produced (those are orders of magnitude according to a unit price index), according to [24].

\begin{tabular}{lll}
\hline Nombre de pièces & Thermoformage & Injection \\
\hline 1000 & 20 & - \\
10000 & 10 & 30 \\
100000 & - & 4 \\
1000000 & - & $<2$ \\
\hline
\end{tabular}

industrie, à l'échelle mondiale, devrait connaître une croissance de $5 \%$ par an jusqu'en 2020 [25]. C'est une méthode qui possède de nombreux avantages comme des temps de cycles courts (quelques secondes), une production possible en grande série (la garantie du moule peut atteindre les deux millions de moulées avec une maintenance adaptée) avec une précision dimensionnelle qui peut atteindre quelques micromètres par l'utilisation de moyens techniques adaptés.

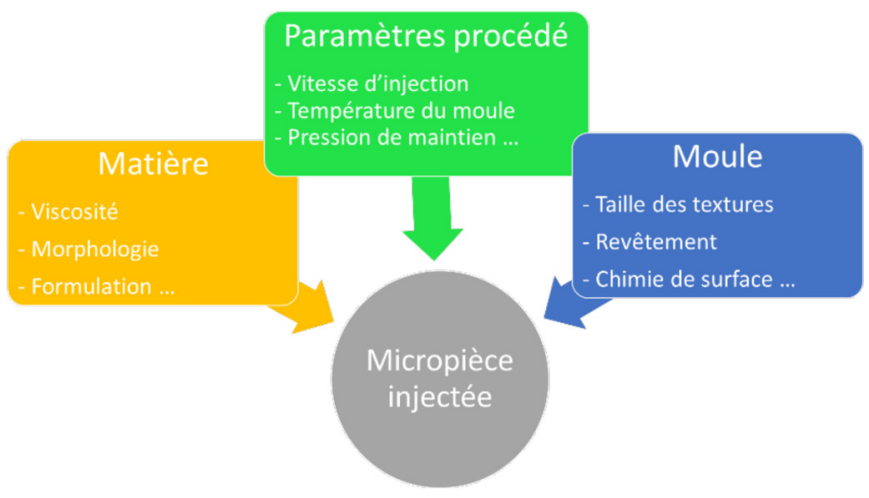

Fig. 1. Facteurs à prendre en compte pour fabriquer par injection une micro/nano-pièce.

Fig 1. Factors that must be considered in microinjection molding.

Les différents éléments qui constituent la presse à injecter, sont présentés sur la figure 2 :

Le cycle d'injection se décompose en 5 étapes (Fig. 3) :

- la phase de dosage : après introduction dans la trémie, les granulés de polymères sont fondus dans un système de 


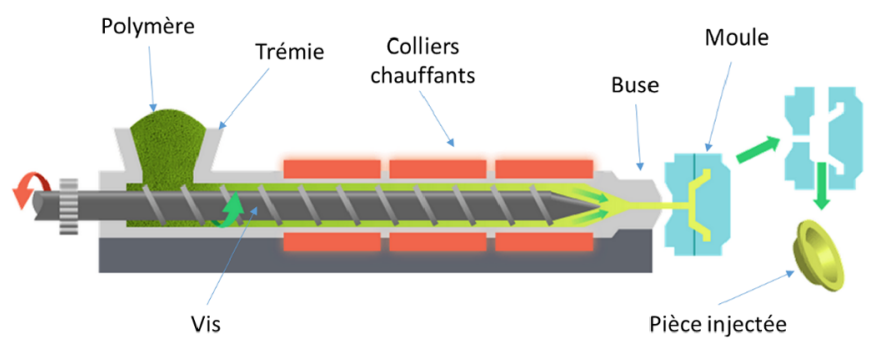

Fig. 2. Schéma de principe d'une presse à injecter, d'après [26].

Fig 2. Schematic diagram of an injection molding machine, according to [26].

a)

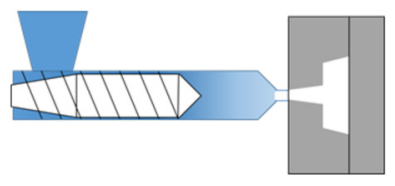

b)

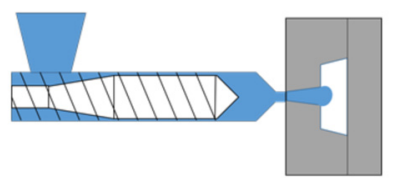

c)

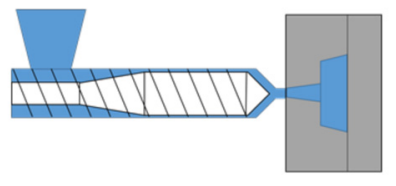

d)

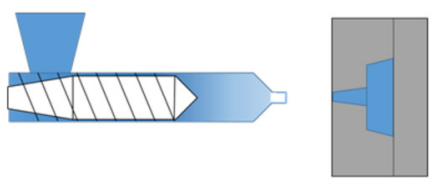

e)

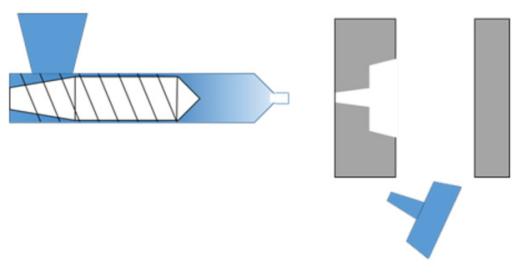

Fig. 3. Étapes du procédé d'injection: (a) départ injection; (b) remplissage; (c) fin de remplissage et phase de maintien; (d) refroidissement et dosage et (e) éjection de la pièce.

Fig 3. Steps of the injection process: (a) injection; (b) filling; (c) end of filling and holding phase; (d) cooling and dosing and (e) ejection of the part.

plastification vis-fourreau, régulé en température, et une dose de matière fondue est accumulée en tête de vis;

- la phase de remplissage dynamique: le polymère fondu est injecté, via la buse d'injection, à vitesse imposée de manière à remplir $99 \%$ de l'empreinte du moule (fermé). La pression d'injection passe par un maximum dit pression de commutation (voir Fig. 4);

- la phase de maintien (ou compactage) : après commutation (passage de la phase de remplissage dynamique en phase de maintien), une pression est imposée sur la vis qui permet un «nourrissement» (apport de matière) de la pièce qui permet la maîtrise du retrait dû au refroidissement de la pièce. Cette phase dure en général jusqu'au refroidissement du seuil à partir duquel aucune pression ne peut ensuite être transmise à la pièce ;

- la phase de refroidissement : le (ou les) seuil(s) d'injection sont entièrement solidifiés. Cette phase correspond au refroidissement de la pièce jusqu'à sa température de démoulage ;

- la phase d'éjection : lorsque la pièce est solidifiée, le moule s'ouvre et la pièce est éjectée hors du moule (avec l'aide d'éjecteurs).

Toutes ces étapes peuvent être suivies in situ par des mesures de pression et de température dans le moule d'injection. La figure 4 présente un exemple d'enregistrement de la pression hydraulique d'injection (pression enregistrée au niveau du vérin qui pilote la vis d'injection) mais aussi des températures et des pressions de la matière mesurées dans l'empreinte.

Lors de la phase de remplissage, l'avancement du front de matière est décrit suivant la figure 5. L'avancée de ce front lors de cette phase a été entièrement décrite par Tadmor en 1974 [27]. Il indique que les vitesses sont légèrement différentes entre le centre de l'écoulement et la surface du moule. L'amplitude de ce gradient de vitesse est liée à la solidification du polymère qui crée une gaine solide qui s'épaissit avec le temps, de la surface du polymère vers le cour. Lorsqu'on regarde la cartographie des vitesses dans le repère lié au front de matière (Fig. 5b), l'effet fontaine apparaît. En effet, le polymère fondu qui est au centre de la gaine va, au contact du front de matière, se diriger sur les bords de la gaine. Cette trajectoire ressemble au jet d'eau d'une fontaine, d'où le nom.

Lorsque la matière a rempli la cavité, on applique une pression de maintien. Cette pression est généralement choisie entre 50 et $80 \%$ de la pression de commutation, Pc.

Enfin, la phase de refroidissement de la pièce est étroitement liée aux températures caractéristiques du polymère $\left(T_{\mathrm{g}}\right.$, température de transition vitreuse du polymère et $T_{\mathrm{f}}$, température de fusion dans le cas de polymères semi-cristallins) et de ses propriétés thermiques. Ainsi, la solidification du polymère a lieu à des temps et des contraintes différents, ce qui induit des morphologies moléculaires complexes des pièces injectées (Fig. 6).

La couche extérieure ou couche de peau, est la partie du polymère fondu est directement en contact avec le moule froid. Elle est le résultat de la solidification rapide du polymère. Selon les auteurs, le nombre de couches distinctes dans l'épaisseur des pièces injectées varient entre 2 et 6 , suivant les méthodes d'analyses utilisées ainsi que le niveau de discrimination arbitrairement choisi. Après la couche de peau vient la zone cisaillée, la zone de post-remplissage et le cœur de la pièce. Ces différentes zones sont très bien décrites par Vite sur un PP injecté [29]. La taille et l'épaisseur des différentes zones sont liées aux différentes conditions d'injection et à la nature du polymère, notamment s'il est amorphe ou semi-cristallin.

Giboz et al. [30] montrent aussi que la microinjection peut avoir une influence sur la pièce fabriquée par rapport à une pièce de taille «standard». En effet, dans des 


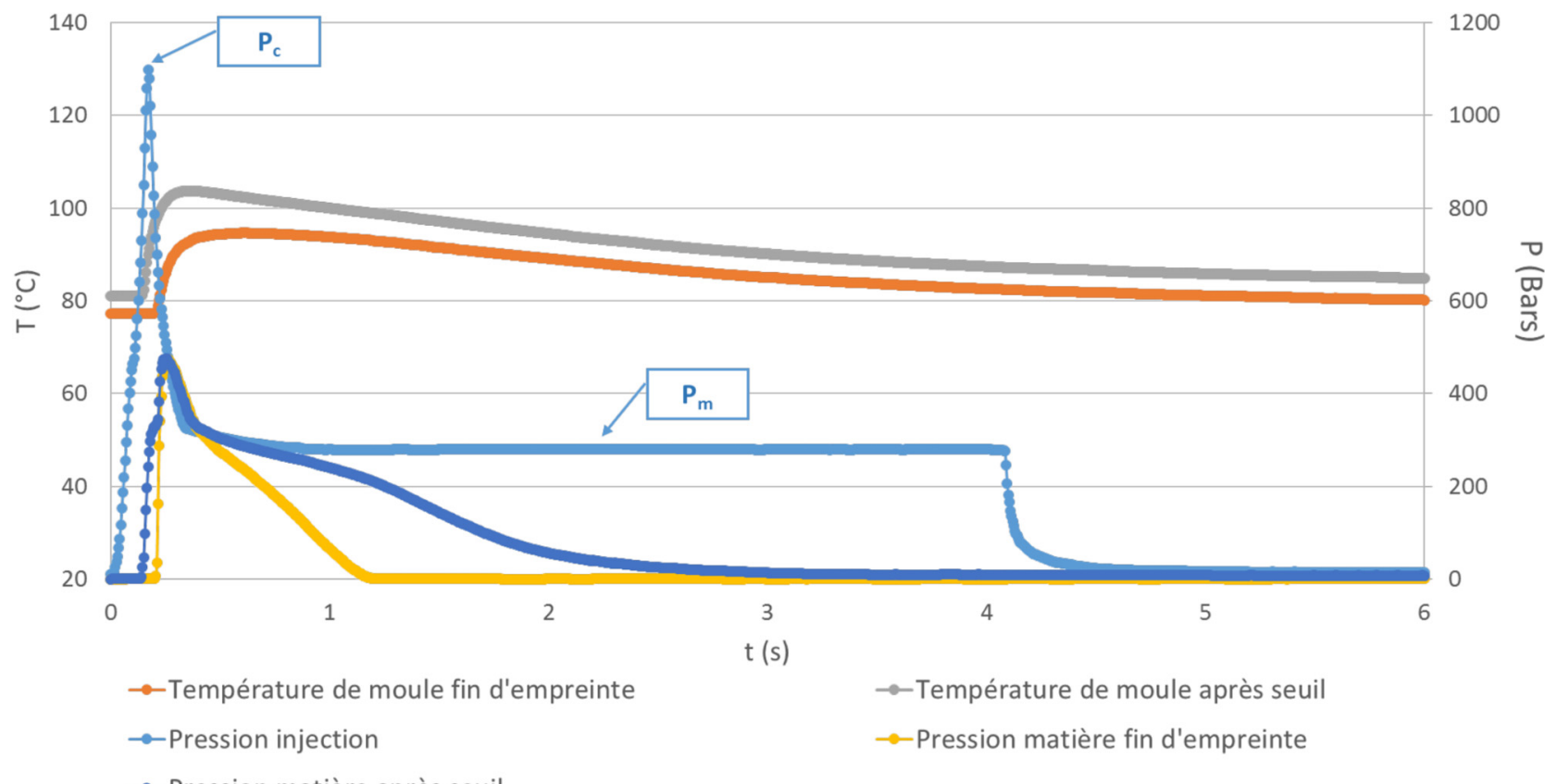

Fig. 4. Courbes obtenues par les différents capteurs lors de la phase de remplissage du moule par du polycarbonate. La pression de commutation, Pc, correspond au maximum de la pression d'injection et la pression de maintien est notée, Pm (voir Sect. 2).

Fig 4. Curves obtained by the various sensors during the phase of filling the mold with polycarbonate. The commutation pressure, Pc, corresponds to the maximum injection pressure and the holding pressure is noted Pm (see Sect. 2).

(a)

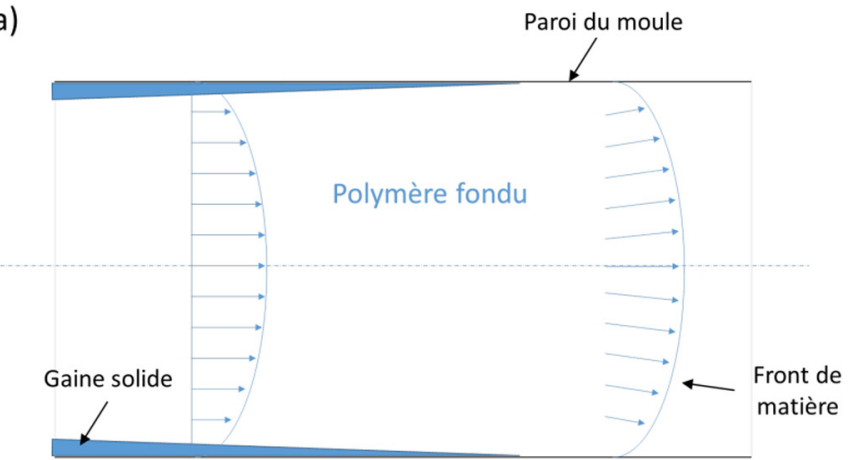

(b)

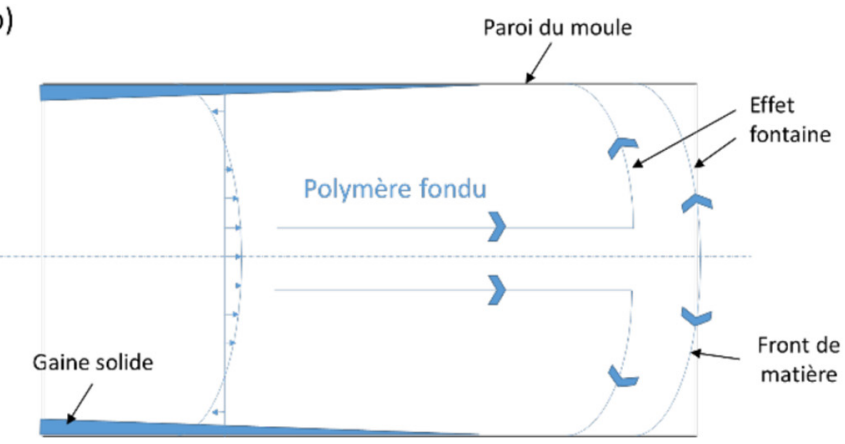

Fig. 5. Champ de vitesses lors du remplissage de la cavité d'un moule: (a) repère fixe, (b) repère lié au front de matière.

Fig 5. Speed field during the filling of the cavity of a mold: (a) fixed marker, (b) mark linked to the material front.

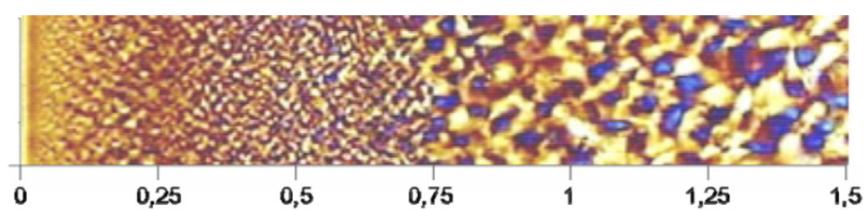

Fig. 6. Micrographie en lumière polarisée de la demi-épaisseur d'une plaque de $3 \mathrm{~mm}$ d'épaisseur injectée en polypropylène, d'après [28] (échelle en $\mathrm{mm}$ ).

Fig 6. Micrograph in polarized light of the half thickness of a $3 \mathrm{~mm}$ thick plate injected in polypropylene, according to [28] (scale in $\mathrm{mm}$ ).

conditions d'injection similaires, une absence de sphérolites a été détectée sur la micro-pièce alors que des sphérolites sont présentes sur la pièce standard. De plus, les différentes couches cristallines sont moins identifiables dans l'épaisseur d'une micro-pièce par rapport à ce que l'on peut observer dans une pièce conventionnelle. Ce résultat peut être dû à des considérations géométriques (part importante du polymère en contact avec le moule apportant ainsi des nucléations hétérogènes ou encore effet important de la cristallisation dû à l'écoulement).

Finalement, le procédé d'injection conditionne la qualité de la pièce injectée et ses différentes caractéristiques. Donc, pour maîtriser au mieux les propriétés de cette pièce (et notamment son état de surface), le premier facteur à prendre en compte est l'ensemble des propriétés liées au polymère utilisé pour fabriquer cette micro-pièce. Les propriétés du polymère en lien avec sa qualité de réplication seront décrites dans le paragraphe suivant. 


\section{Matière}

Les polymères sont un enchaînement de plusieurs monomères liés les uns aux autres. Ils peuvent être assimilés à de très grandes molécules, on parle alors de macromolécules. La compréhension des propriétés du polymère demande la connaissance de la constitution de cette macromolécule (configuration, conformation, longueur). La longueur de la chaîne macromoléculaire est notamment un paramètre influant fortement sur les propriétés macroscopiques. Cette longueur est souvent exprimée en termes de poids moléculaire de la chaîne, liée à la masse moléculaire du monomère et du nombre de monomères connectés dans une chaîne. Cependant, les polymères synthétiques sont polydisperses et contiennent des chaînes de différentes longueurs. On définit alors un indice de polymolécularité (IP) tel que IP $=M_{\mathrm{n}} / M_{\mathrm{w}}$ qui traduit la distribution des masses molaires dans le polymère. La définition d'un polymère nécessite donc de déterminer sa masse molaire moyenne en masse $\left(M_{\mathrm{w}}\right)$, sa masse molaire moyenne en nombre (qui correspond à la moyenne des masses molaires pondérée par le nombre de chaînes de chaque longueur) et la distribution de ces masses moyennes, comme montré sur la figure 7 .

Dans notre étude, nous nous intéresserons principalement aux thermoplastiques qui sont les polymères les plus souvent mis en forme par injection. En fonction de leur structure moléculaire et des conditions de mise en œuvre, ils se présentent à l'état solide sous une forme amorphe ou semi-cristalline. Les polymères commerciaux, et plus particulièrement les polyoléfines, sont disponibles avec différents grades (ou viscosités à l'état fondu). Ainsi le nombre IP renseigne sur l'homogénéité du polymère alors que la viscosité à l'état fondu est fonction du $M_{\mathrm{w}}$. Plus $M_{\mathrm{w}}$ est élevée, plus les chaînes polymériques sont longues et plus généralement le polymère à l'état fondu est visqueux [31,32].

Les thermoplastiques sont caractérisés par un début de ramollissement lors du chauffage lorsque leur température dépasse environ 0,75 fois leur température de transition vitreuse $\left(T_{\mathrm{g}}\right)$ et une solidification lors du refroidissement. Ainsi, on choisira une température de fourreau (ou température d'injection) au moins $100^{\circ} \mathrm{C}$ au-dessus de cette valeur, selon la stabilité thermique du polymère. Pour les polymères semi-cristallins, il existe également une température de fusion $\left(T_{\mathrm{f}}\right)$. On fixe alors la température de fourreau environ $50{ }^{\circ} \mathrm{C}$ au-dessus de cette $T_{\mathrm{f}}$. Le chauffage d'un polymère thermoplastique jusqu'à son état fondu peut-être répété et son comportement est réversible. Cependant, il peut se dégrader si la température dépasse un certain niveau pour lequel les liaisons entre les groupes monomères sont rompues.

Une des clés de la qualité des pièces produites en polymère, résulte dans l'adaptation des paramètres du procédé aux caractéristiques spécifiques de chaque polymère, d'une part et à la géométrie de la pièce ainsi qu'un ensemble de caractéristiques propres au procédé (emplacement, nombre de points d'injection, cycle de moulage...) d'autre part. Il est notamment particulièrement important d'adapter les températures caractéristiques du procédé

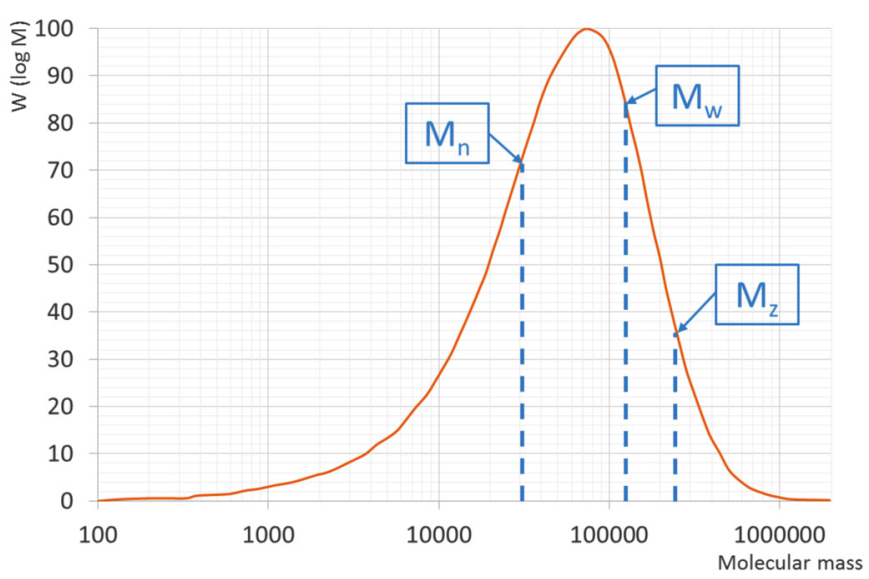

Fig. 7. Exemple de distribution des masses molaires d'un polypropylène.

Fig 7. Example of distribution of the molar masses of a polypropylene.

Tableau 4. Principales lois pour modéliser la viscosité du polymère en fonction de la viscosité et/ou la température.

Table 4. Main laws to model the viscosity of the polymer as a function of viscosity and/or temperature.

\begin{tabular}{lll}
\hline Modèles & Lois rhéologiques & Références \\
\hline Cross & $\eta(\dot{\gamma})=\frac{\eta_{0}}{(1+\lambda \dot{\gamma})^{1-m}}$ & {$[33]$} \\
Carreau & $\eta(T, \dot{\Upsilon})=\frac{\eta_{0}(T)}{\left[1+\left(\frac{\eta_{0}(T)}{\tau} \dot{\Upsilon}\right)^{2}\right]^{\frac{1-m}{a}}}$ & {$[34]$} \\
Carreau-Yasuda & $\eta(T, \dot{\Upsilon})=\frac{\eta_{0}(T)}{\left[1+\left(\frac{\eta_{0}(T)}{\tau} \dot{\Upsilon}\right)^{a}\right]^{\frac{1-m}{a}}}$ & {$[35]$} \\
\hline
\end{tabular}

(température du fourreau, $T_{\mathrm{fo}}$, température du moule, $\left.T_{\mathrm{m}} \ldots\right)$ en fonction de celles propres au polymère $\left(T_{\mathrm{g}}, T_{\mathrm{f}} \ldots\right)$. En effet, il est bien connu que la viscosité des polymères fondus est dépendante du taux de cisaillement et de la température, ainsi la modification de la température du fourreau ou de la vitesse d'injection modifie la rhéologie du polymère et induit un effet couplé sur la matière.

On considère généralement que la variation de viscosité obéit à la loi de William-Landel-Ferry (WLF) à faible température. À haute température, les polymères fondus ont un comportement pseudoplastique et leur viscosité diminue avec l'augmentation du taux de cisaillement. C'est pourquoi, pour des températures supérieures à la $T_{\mathrm{g}}$, comme celles présentes lors du procédé d'injection, différentes expressions ont été proposées pour corréler la viscosité, $\eta$, avec le taux de cisaillement $\dot{\gamma}$. Les expressions les plus connues sont présentes dans le tableau 4 .

Le choix d'une loi plutôt qu'une autre dépend de la nature du polymère [36]. De plus, lors de l'injection, le polymère s'écoule dans une cavité plus ou moins complexe. Ainsi, la modélisation de la rhéologie du polymère dépendra 
Tableau 5. Liste non exhaustive de polymères utilisés en micro injection (SC: semi-cristallin; A : amorphe, $T_{\mathrm{g}}$ : température de transition vitreuse; $T_{\mathrm{f}}$ : température de fusion. Les valeurs en italique sont des valeurs génériques.

Table 5. Non-exhaustive list of polymers used in microinjection (SC: semi-crystalline, A: amorphous, $T_{g}$ : glass transition temperature, $T_{f}$ : melting temperature, italic values are generic values).

\begin{tabular}{|c|c|c|c|c|c|c|c|}
\hline Morphologie & Matériau & $\begin{array}{l}\text { Viscosité (MFI) } \\
(\mathrm{g} / 10 \mathrm{~min})\end{array}$ & $\begin{array}{l}T_{g} \\
\left({ }^{\circ} \mathrm{C}\right)\end{array}$ & $\begin{array}{l}T_{\mathrm{f}} \\
\left({ }^{\circ} \mathrm{C}\right)\end{array}$ & $\begin{array}{l}\text { Température d'injection } \\
\left({ }^{\circ} \mathrm{C}\right)\end{array}$ & $\begin{array}{l}\text { Retrait } \\
(\%)\end{array}$ & Références \\
\hline \multirow{4}{*}{$\mathrm{SC}$} & HDPE & $\begin{array}{l}25 \\
\left(190{ }^{\circ} \mathrm{C} / 2,16 \mathrm{~kg}\right)\end{array}$ & $128-135$ & 132 & $200-210$ & 1,3 à 3 & {$[42-44]$} \\
\hline & $\mathrm{PBT}$ & $\begin{array}{l}19 \\
\left(250{ }^{\circ} \mathrm{C} / 2,16 \mathrm{~kg}\right)\end{array}$ & 60 & 225 & $290-325$ & 1 à 2 & {$[45]$} \\
\hline & POM & - & -65 & $160-170$ & 210 & 2 à 2,5 & {$[46]$} \\
\hline & $\mathrm{PP}$ & $\begin{array}{l}7 \\
\left(230{ }^{\circ} \mathrm{C} / 2,16 \mathrm{~kg}\right)\end{array}$ & -10 & 160 & $205-260$ & 0,7 à 2,5 & {$[47]$} \\
\hline \multirow{4}{*}{ A } & $\mathrm{PC}$ & $\begin{array}{l}10 \text { et } 63 \\
\left(300^{\circ} \mathrm{C} / 2,16 \mathrm{~kg}\right)\end{array}$ & 145 & - & $260-300$ & 0,7 à 0,8 & {$[47-49]$} \\
\hline & PS & $\begin{array}{l}14 \\
\left(200^{\circ} \mathrm{C} / 5 \mathrm{~kg}\right)\end{array}$ & 90 & - & 205 & 0,2 à 0,6 & {$[50,51]$} \\
\hline & $\mathrm{COC}$ & $\left(260{ }^{\circ} \mathrm{C} / 2,16 \mathrm{~kg}\right)$ & 140 & - & $290-310$ & 0,1 à 0,7 & {$[47]$} \\
\hline & PMMA & $\begin{array}{l}6 \\
\left(230^{\circ} \mathrm{C} / 5 \mathrm{~kg}\right)\end{array}$ & 100 & - & $210-140$ & 0,2 à 0,6 & {$[47]$} \\
\hline
\end{tabular}

de la cavité à remplir ainsi que des canaux d'alimentation. D'un point de vue industriel, la viscosité des polymères est souvent caractérisée par leur grade MFI (voir Tab. 5) [37]. Cette indice traduit une viscosité sous faible vitesse de cisaillement (quelques $\mathrm{s}^{-1}$ ) alors que les injections se réalisent parfois à des valeurs supérieures à $10^{5} \mathrm{~s}^{-1}$, il est donc particulièrement important de connaître ce comportement dans les conditions réelles d'injection, ce d'autant plus que certains polymères de MFI très différents peuvent avoir une viscosité relativement proche pour des vitesses de cisaillement élevées [38]. Ainsi, les études rhéologiques en lien avec la taille des structures à remplir se multiplient pour répondre aux problématiques de remplissage de microcavités $[39,40]$.

Un autre point important est la phase de refroidissement du polymère dans les empreintes. Cette phase conditionne le retrait, en fonction notamment des paramètres morphologiques du polymère (nature et structure du polymère, présence de charges...). Le tableau 5 donne des valeurs moyennes de retrait pour des polymères appartenant à différentes familles. Les fortes amplitudes données dans certains cas (HDPE ou PP) montrent l'évolution possible des valeurs en fonction des conditions de mise en œuvre ou de la direction considérée pour la mesure. Elles mettent en évidence une différence de comportement entre les polymères amorphes (retrait $<1$ $\%$ ) et les polymères semi-cristallins (retrait $>1 \%$ ). L'origine de cette différence est liée à la morphologie et à l'organisation moléculaire qui caractérise ces deux états. L'état amorphe ne présente aucun arrangement périodique à longue distance des macromolécules entre elles. À l'état relaxé, une molécule se trouve dans sa configuration la plus stable: la pelote statistique. Le passage de l'état fondu à l'état vitreux (lors du passage de la $T_{\mathrm{g}}$ ) s'accompagne d'une faible variation du volume spécifique, donc un faible retrait.

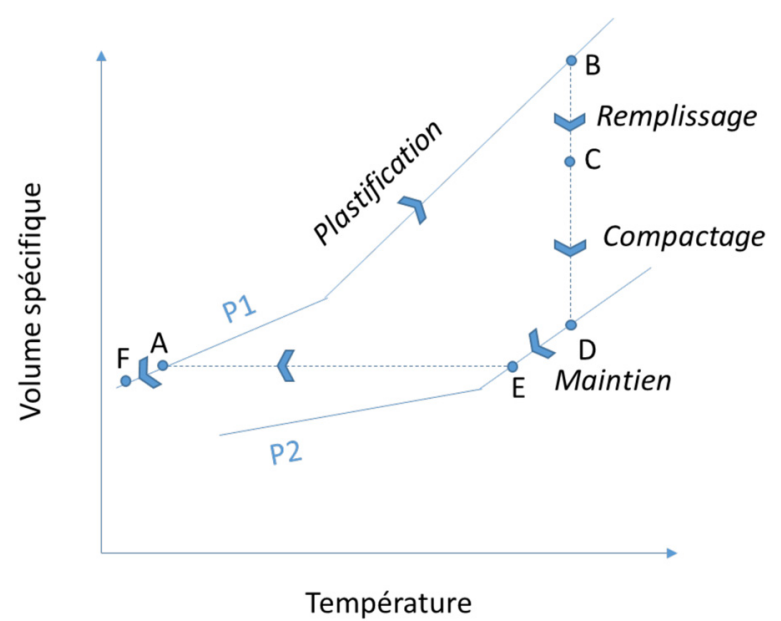

Fig. 8. À partir du diagramme PVT d'un polymère amorphe, schéma du trajet virtuel décrit par ce polymère durant un cycle d'injection, d'après [41]. P1 et P2 sont deux pressions auxquelles sont soumis le polymère dans les phases considérées, typiquement $\mathrm{P} 1$ est de l'ordre du dixième de MPa tandis que P2 est de l'ordre de la dizaine de MPa.

Fig 8. From the PVT diagram of an amorphous polymer, a diagram of the virtual pathway described by this polymer during an injection cycle, according to [41]. P1 and P2 are two pressures to which the polymer is subjected in the phases under consideration, typically P1 is of the order of one tenth of MPa while P2 is of the order of ten of $M P a$.

La figure 8 présente cette évolution du volume spécifique en fonction des différentes étapes du cycle d'injection en tenant compte du chauffage et du refroidissement du polymère amorphe ainsi que de la pression à laquelle il est soumis. 
L'état cristallin correspond à des arrangements locaux des chaînes macromoléculaires qui constituent des lamelles cristallines avec des phases amorphes occupant les espaces inter-lamellaires. Lors du refroidissement, le passage du polymère de l'état fondu (amorphe) à l'état solide cristallisé (semi-cristallin) s'accompagne d'une importante variation du volume spécifique, d'où un retrait important, sans passer la $T_{\mathrm{g}}$. On imagine aisément que le retrait a une incidence sur la qualité de reproduction des nanostructures mais il est compliqué de quantifier ce phénomène. Le tableau 5 répertorie (de manière non-exhaustive) les polymères les plus utilisés dans la réplication de textures submicroniques.

Dans la littérature, on trouve des études sur une multitude de polymères avec lesquels on reproduit des nanostructures. Les températures d'injection utilisées pour la microinjection sont équivalentes à celles utilisées en injection conventionnelle en revanche les vitesses de cisaillement peuvent être très supérieures. Ainsi, des conditions spécifiques doivent être choisies pour une bonne réplication des pièces.

La caractérisation du polymère injecté est l'une des clés pour une réplication de qualité. Certaines caractéristiques conventionnelles (viscosité à partir du MFI) doivent être utilisées avec discernement quand les vitesses de cisaillement en microinjection peuvent être supérieures à $10^{5} \mathrm{~s}^{-1}$ et des vitesses de solidification supérieures à $100 \mathrm{~K} / \mathrm{s}$. Les paramètres d'injection influent de façon très importante sur le type de cristallisation obtenue au sein d'une micropièce et cela impacte fortement les caractéristiques mécaniques du matériau. En effet, les faibles épaisseurs des cavités en microinjection génèrent l'augmentation de l'orientation moléculaire [52] puisque la relaxation des chaînes de polymères est freinée par les taux de cisaillement et les vitesses de refroidissement plus importantes. Pour les polymères semi-cristallins, ces conditions sont déjà connues pour engendrer des orientations moléculaires importantes et des structures cristallines telles que des «shish-kebabs» qui induisent des variations importantes des propriétés mécaniques [53].

On a vu que la qualité de réplication dépend du polymère et de ses différentes propriétés. Dans le procédé de microinjection, la composition du moule influence aussi la qualité de la pièce injectée. C'est ce que nous allons voir dans le paragraphe suivant.

\section{Moule}

Pour ces propriétés mécaniques, thermiques et son usinabilité, les moules industriels employés sur les presses à injecter sont encore principalement à base d'acier. Sur ce type d'outil, il faut distinguer l'acier de la carcasse de celui des empreintes (moulantes). Pendant longtemps les développements de nouveaux aciers pour ces empreintes ou bien leurs traitements de surface, avaient pour objectif d'augmenter leur durée de vie en privilégiant les propriétés de dureté et de résistance à chaud. Ainsi les aciers à moule étaient la plupart du temps des aciers inoxydables ou des aciers à coupe rapide avec une dureté HRC variant de 40 à 65 [54]. Toutefois la prise en compte de la résistance aux chocs et à la fatigue thermodynamique ont conduit les sidérurgistes à proposer de nouvelles nuances avec des teneurs réduites en manganèse, $\mathrm{Mn}$, et silicium, Si. L'incorporation d'éléments d'alliages très oxydables comme l'aluminium et le cuivre dans des aciers contenant $3 \%$ de nickel et un faible pourcentage de carbone permettent d'avoir des niveaux de résistance au moins comparables à ceux des aciers prétraités comme le 40CrMnMo8. On obtient avec ces aciers un gain de $20 \%$ en conductivité thermique tout en réduisant le nombre de ségrégation d'éléments d'alliages ainsi qu'une meilleure aptitude au grainage et au polissage conduisant in fine, à de bien meilleurs états de surface [55].

\subsection{Revêtements des empreintes}

Les revêtements sur les moules de plasturgie ont été étudiés depuis les années 1980 souvent pour améliorer la durabilité des outils [56] ou bien limiter l'adhésion du polymère à l'acier au moment du démoulage. Cette étape supplémentaire dans la fabrication de l'outil introduit un surcoût à la base mais peut être amortie par la quantité de pièces plastiques produites. Pour une meilleure réplication de ces pièces à partir d'un moule revêtu, Saha et al. [57] propose, pour le revêtement, le cahier des charges suivant:

- avoir un faible coefficient de friction;

- avoir une haute résistance à l'usure notamment abrasive (particulièrement marquée pour les polymères chargés de

fibres de verre...);

- être chimiquement inerte;

- être stable thermiquement;

- pouvoir supporter des hautes pressions ;

- bien adhérer aux matériaux de base;

- et pouvoir remplir toutes ces fonctions même avec une épaisseur de quelques nanomètres.

On peut aussi ajouter que le dépôt doit être uniformément réparti sur toute la zone moulante et que l'état de surface doit pouvoir être maitrisé aux différentes échelles en fonction du cahier des charges.

Ainsi les revêtements anti-usures pour outils à base classiquement de nitrures métalliques ou DLC (Diamond Like Carbon) on fait l'objet de nombreux travaux [58,59]. Les procédés mise en ouvre pour leur élaboration sur quelques micromètres d'épaisseur, sont souvent des procédés par voie sèche comme la PVD (Physical Vapor Deposition) ou la CVD (Chemical Vapor Deposition).

\subsubsection{Dépôts CVD et PACVD}

En CVD ou par voie chimique en phase vapeur, le matériau source est inséré sous forme gazeuse dans l'enceinte de dépôt. En fonction des conditions thermodynamiques (températures élevées du substrat, pression) le gaz précurseur réagit chimiquement à la surface du substrat conduisant à la croissance du dépôt de manière relativement homogène, n'étant pas influencé par la topographie de surface [73]. Dans le cas de la PACVD (Plasma Assisted Chemical Vapor Deposition) l'énergie apportée pour provoquer la dissociation des molécules du gaz précurseur provient d'un plasma créé dans l'enceinte de dépôt. La 
formation de nombreux radicaux augmente ainsi la réactivité des précurseurs. Les vitesses de dépôt sont alors plus élevées ou de manière équivalente, les températures de dépôts peuvent être diminuées par rapport aux conditions équivalentes de CVD. Le fait d'utiliser des aciers à outils ayant préalablement subis un traitement thermique, limite les températures de dépôt le plus souvent à des valeurs inférieures à $400^{\circ} \mathrm{C}$, ce qui conduit à généralement privilégier les procédés PACVD.

À partir de précurseurs de type hydrocarbures tels que du $\mathrm{CH}_{4}, \mathrm{C}_{2} \mathrm{H}_{2}$ additionnés d'hydrogène, sont réalisés des dépôts de DLC par PACVD. Très largement étudiés pour leur très faible coefficient de frottement avec l'acier (souvent inférieur à 0,1) [59] et leur bonne résistance à l'usure associée à une dureté, $H$, élevée $(H$ souvent $>20$ $\mathrm{GPa}$ ) pour des températures inférieures à $300^{\circ} \mathrm{C}$, ces dépôts ont montrés une certaine efficacité en injection [60].

\subsubsection{Dépôts PVD}

Les dépôts par voie physique en phase vapeur, s'effectuent à basse pression dans une enceinte sous vide dans laquelle est introduit un gaz neutre et/ou réactif à partir duquel un plasma froid est créé. Le transfert de matière de la cible solide vers le substrat est provoqué par le bombardement d'ions énergétiques générés dans le plasma qui induisent des cascades de déplacements au sein de la cible dont le résultat est l'émission de particules. Ce flux de matière condensable, se dépose sur le substrat et permet la croissance de la couche. Le caractère directionnel et énergétique de ce flux de particules a notamment pour effet de créer des phénomènes d'ombrage ou d'inhomogénéité de vitesses de croissance des dépôts en fonction de la géométrie de l'installation et de celle des substrats ainsi que des contraintes internes dans le revêtement pouvant être importantes (supérieures au GPa dans les nitrures) même si les températures de dépôt sont souvent inférieures à $300^{\circ} \mathrm{C}$. Des solutions technologiques de compromis existent (cinématique particulière des substrats pendant le dépôt, polarisation du substrat...) mais la conformité des revêtements et des motifs en texturation est un point toujours délicat. Néanmoins, cette technique, largement décrite dans la littérature [61-64] est la plus utilisée industriellement pour le dépôt de films minces notamment de nitrures métalliques.

En plasturgie, de nombreux travaux décrivent l'intérêt de revêtir le moule pour augmenter sa durabilité. Un revêtement de nitrure de titane, TiN, ou de nitrure de chrome, $\mathrm{CrN}$, permet à un moule d'avoir une durée de vie multipliée par deux ainsi qu'une réduction du temps de cycle tout en améliorant l'état de surface [55]. De même après avoir injecté 250 moulées de PEHD, Neto et al. concluent que leur revêtement DLC (Diamond Like Carbon) n'a subi aucune détérioration [65]. Ce même intérêt pour les revêtements DLC en termes de durabilité du moule et de résistance à la rayure est présenté par Loan et al. [54]. Ils démontrent qu'un revêtement DLC sur un outillage d'injection lui confère une excellente protection contre les rayures et les endommagements [66]. Cunha et al. $[67,68]$ expliquent aussi qu'un revêtement PVD sur l'acier du moule peut diminuer le volume d'usure par kilogramme

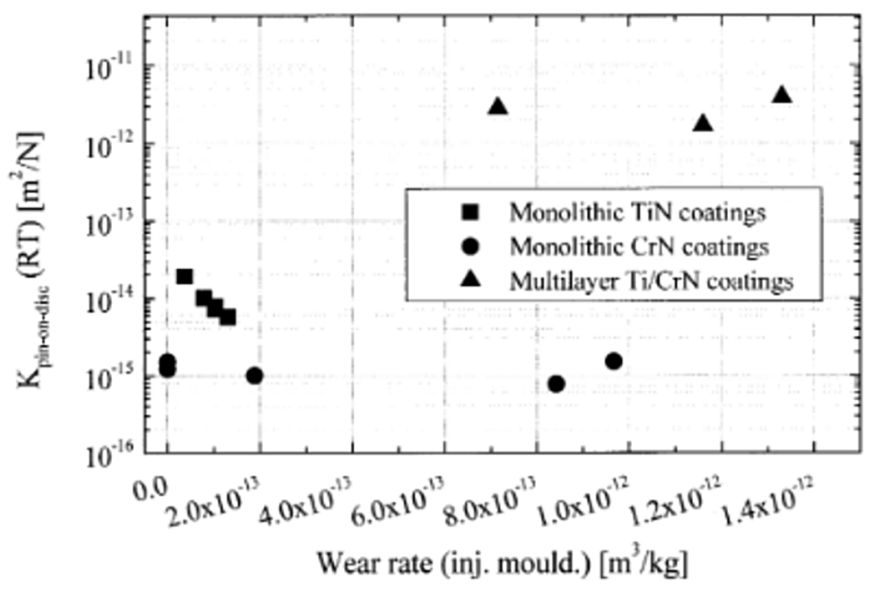

Fig. 9. Relation entre l'usure induite par les cycles d'injection (Wear rate (inj. Mould.)) et l'usure par essais pion/disque, d'après [67]. Reproduit avec la permission de l'éditeur Elsevier.

Fig 9. Relationship between wear induced by injection cycles (Wear rate (injection mold)) and wear by pin/disc test, according to [67].

de polymère injecté d'un facteur cent par rapport à l'acier $\mathrm{nu}$ (indépendamment des traitements thermiques) et qu'il apporte une très bonne protection à la corrosion vis-à-vis de l'injection de PA6.6 renforcé par des fibres de verre.

Ainsi on peut admettre que revêtir un moule d'un dépôt dur et adhérant augmente sa résistance à l'usure et prolonge sa durée de vie $[67,69]$. Sur la base de ce type d'observation Bull et al. proposent de sélectionner les traitements de surface pour des outillages d'injection (vis...) à partir d'essais tribologiques dérivés de ceux utilisés pour caractériser l'usure abrasive [70], tandis que Cunha et al. partent d'un essai pion-disque (un pion frottant contre un disque en rotation) effectué à $300^{\circ}$ [67]. Ces derniers (Fig. 9) montrent qu'il n'y a pas de corrélation entre ces résultats d'usure issus de l'essai pion-disque et l'usure induite par les cycles d'injection. En effet, le revêtement $\mathrm{CrN}$ (faible usure pion-disque) a une usure plus prononcée que le TiN pour un usage fonctionnel du moule.

Dans le cadre de la microinjection, le ratio de la surface développée sur le volume de la partie moulante de l'outil est très élevé. Les propriétés de surface comme l'adhésion et la friction durant la phase de démoulage jouent donc un rôle important où l'apport d'un revêtement a tout son intérêt. Chailly et al. montrent que les revêtements peuvent réduire les défauts de pièces ou encore diminuer les forces d'éjection et éviter une déformation des textures répliquées lors du démoulage [60]. Le tableau 6 regroupe une liste non exhaustive des revêtements utilisés lors de réplication de surfaces submicroniques par injection.

Ainsi, si l'on cherche à sélectionner des revêtements afin d'améliorer les conditions d'injection, cela nécessite de dissocier leurs différents effets sur la durabilité du moule (usures abrasives, adhésives, corrosion...) d'une part, de la qualité de la pièce injectée, d'autre part. Cela signifie aussi de quantifier séparément l'usure de l'outillage, de l'adhésion polymère-moule. Cette adhésion a notamment été déterminée par des mesures de forces de friction polymère- 
Tableau 6. Liste (non exhaustive) de revêtements utilisés dans la réplication de surfaces nano-structurées par injection.

Table 6. List (not exhaustive) of coatings used in the replication of nano-structured injection surfaces.

\begin{tabular}{|c|c|c|c|c|c|}
\hline Revêtement & Substrat & $\begin{array}{l}\text { Polymère } \\
\text { injecté }\end{array}$ & Performance & $\begin{array}{l}\text { Rapport } \\
\text { de forme }\end{array}$ & Références \\
\hline $\begin{array}{l}\text { FOTS } \\
\text { (trichloro- }[1 \mathrm{H}, 1 \mathrm{H},\end{array}$ & $\mathrm{Si}$ & $\mathrm{TPU}$ & $\begin{array}{l}\text { Protection du moule et facilite le } \\
\text { remplissage des trous }\end{array}$ & 2,3 & {$[71]$} \\
\hline $\begin{array}{l}2 \mathrm{H}, 2 \mathrm{H}- \\
\text { perfluorooctyl|-silane }\end{array}$ & $\mathrm{Ni}$ & $\mathrm{COC}$ & Aide au remplissage de trous de $100 \mathrm{~nm}$ & $>2,5$ & {$[72]$} \\
\hline FS, MoN, WN & $\mathrm{Ni}$ & PC, PMP & $\begin{array}{l}\text { Revêtement anti-adhésif qui prévient de } \\
\text { l'accumulation de polymères sur } 1500 \\
\text { cycles avec un réseau de } 0,2-1 \mu \mathrm{m}\end{array}$ & 4,5 & {$[69]$} \\
\hline $\mathrm{SiOC}, \mathrm{DLC}$ & Cuivre & $\mathrm{PC}, \mathrm{ABS}$ & $\begin{array}{l}\text { Réduction des forces de démoulage de } \\
40 \%\end{array}$ & 1 & {$[73]$} \\
\hline $\mathrm{CrN}, \mathrm{TiN}$ & Acier & $\begin{array}{l}\text { PA avec fibres } \\
\text { de verre }\end{array}$ & $\begin{array}{l}\text { Faible usure par rapport à des méthodes } \\
\text { classiques (traitements thermique, dépôt } \\
\text { de chrome ou nitruration) }\end{array}$ & - & {$[67]$} \\
\hline $\begin{array}{l}\text { HSQ } \\
\text { (hydrogen } \\
\text { silsesquioxane) }\end{array}$ & Acier & $\mathrm{COC}$ & $\begin{array}{l}\text { Meilleure uniformité de la réplication des } \\
\text { piliers qu'avec un moule en acier } \\
\text { Retarde le refroidissement du polymère } \\
\text { durant la phase de remplissage grâce à } \\
\text { sa faible conductivité thermique }\end{array}$ & $>1$ & {$[74]$} \\
\hline
\end{tabular}

outil soit ex situ à partir d'équipements spécifiques de laboratoire [75], soit in situ dans le moule en équipant un éjecteur d'un capteur de force [76]. Peter Jones [77], dans un procédé d'injection classique, propose de prendre en compte le coefficient de friction, les propriétés mécaniques du polymère, le retrait et les dimensions de la pièce pour calculer la force nécessaire à l'éjection de la pièce, $F_{\text {é }}$, telle que :

$$
F_{\text {é }}=\frac{E \times A \times \mu \times \alpha \times \triangle T}{\frac{d}{2 e}\left(1-\frac{v}{2}\right)},
$$

avec, $A$ : surface de contact de la pièce sur le noyau ou l'empreinte du moule, $\mu$ : coefficient de friction, $\alpha$ : CTE du polymère, $\Delta T$ : différence entre la température de ramollissement du polymère et du moule, $D$ : diamètre du noyau, $e$ : épaisseur, $v$ : coefficient de poisson du polymère. Charmeau et al. [76] montrent qu'un revêtement en TiN ou CrN, comparé à l'acier, permet de diviser par deux le coefficient de friction lors de l'étape de démoulage (en passant de 0,015 pour un acier nu à 0,007 pour un revêtement à base de chrome). Toutefois ce coefficient de frottement est mesuré à partir d'une force de frottement liée au glissement de la pièce sur le moule lors de l'éjection. Si ce coefficient prend en compte le travail d'adhésion polymère-moule, il intègre aussi différents aspects dissipatifs se produisant durant cette étape de séparation des éléments comme un essai d'adhérence classique. Des travaux récents concentrent leur analyse plus particulièrement sur l'interaction physico-chimique entre le polymère fondu et le moule.

Chen et al. [78] montrent que la température et la viscosité du polymère jouent un rôle important dans l'adhésion du polymère sur le moule. Ainsi, plus la vitesse d'injection est élevée, plus l'adhésion entre la pièce et le moule est importante. Cependant, cette force d'adhésion est difficile à mesurer lors du procédé d'injection et demande une conception particulière du moule. C'est pourquoi, certains auteurs déterminent l'adhésion entre le polymère fondu et le moule ex situ. En comparant, l'étalement de quatre polymères fondus sur quatre substrats différenciés par leur rugosité, Zhang et al. constatent le rôle important de la rugosité et de la température dans le procédé de microinjection [79]. D'un autre côté, Bagcivan et al. [80] ont étudié la capacité d'adhésion de deux polymères sur différents dépôts. Ils montrent que le choix des revêtements est important dans l'étalement du polymère. Zitzenbacher et al. [81], proposent des équations semi-empiriques de l'angle de contact des granulés de polymère fondus avec le solide revêtu en fonction de la tension de surface du revêtement telle que:

$$
\begin{gathered}
\text { HDPE }: \theta=81,7-0,4 \gamma_{s}, \\
\text { PP }: \theta=89,6-0,3 \gamma_{s}, \\
\text { PMMA : } \theta=89,8-0,5 \gamma_{s}, \\
\text { PA6.6 }: \theta=107,7-0,7 \gamma_{s},
\end{gathered}
$$

avec, $\gamma_{s}$ : tension de surface du solide à $25^{\circ} \mathrm{en} \mathrm{mJ} / \mathrm{m}^{2}$ et $\theta$ en $\left(^{\circ}\right)$ : l'angle de contact entre le polymère fondu et le substrat.

Il faut noter que les tensions de surface des différents substrats ont été déterminées à $25^{\circ} \mathrm{C}$ et les essais de fonte $\mathrm{du}$ polymère sur les différents substrats se font à des températures de mise en œuvre du polymère (par exemple $200{ }^{\circ} \mathrm{C}$ pour le PP). Ils concluent que pour avoir une faible 

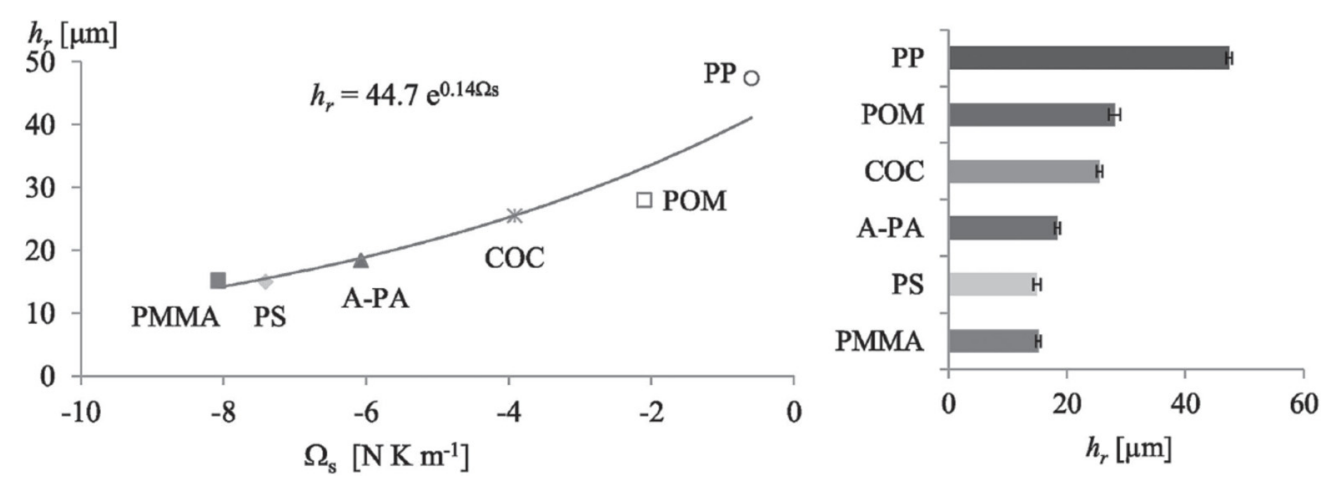

Fig. 10. Hauteur des répliques $\left(h_{\mathrm{r}}\right)$ en fonction du potentiel de démouillage $\left(\Omega_{\mathrm{S}}\right)$, d'après [82]. Reproduit avec la permission de l'éditeur John Wiley and Sons.

Fig 10. Height of the replicas $\left(h_{r}\right)$ as a function of the dewetting potential ( $\Omega$ s), according to [82].

Tableau 7. Procédés d'usinage submicroniques de moule d'injection.

Table 7. Submicron machining processes of injection mold.

\begin{tabular}{|c|c|c|c|c|c|}
\hline Procédés & & $\begin{array}{l}\text { Dimensions } \\
\text { typiques des } \\
\text { structures } \\
(\mu \mathrm{m})\end{array}$ & $\begin{array}{l}\text { Rapport max } \\
\text { hauteur/ } \\
\text { largeur des } \\
\text { structures }\end{array}$ & $\begin{array}{l}\text { Matériaux } \\
\text { typiques de } \\
\text { l'empreinte }\end{array}$ & Références \\
\hline \multirow{3}{*}{ Lithographie } & $\begin{array}{l}\text { Lithographie UV (ou } \\
\text { photolithographie) et } \\
\text { électroformage par galvanisation }\end{array}$ & 0,03 à 500 & 1 à 10 & $\begin{array}{l}\text { Nickel et alliages } \\
\text { de nickel }\end{array}$ & [84-86] \\
\hline & $\begin{array}{l}\text { Lithographie par faisceau } \\
\text { d'électrons et électroformage par } \\
\text { galvanisation }\end{array}$ & 0,1 à 0,5 & 1 & $\begin{array}{l}\text { Nickel et alliages } \\
\text { de nickel }\end{array}$ & {$[47,84,85]$} \\
\hline & Soft lithographie & 0,3 & 5 & & {$[87,88]$} \\
\hline Laser & Laser & 0,6 à 25 & 10 & Métal & {$[86,89]$} \\
\hline
\end{tabular}

adhésion du polymère fondu sur un substrat, il faudrait avoir un substrat avec une rugosité importante et une faible énergie de surface tout en ne négligeant pas la tension superficielle du polymère fondu. En revanche, aucun de ces essais n'est directement corrélé avec une application sur un procédé de mise en œuvre du polymère comme des essais de microinjection, par exemple.

Rytka et al. [82] comparent, quant à eux, les valeurs d'étalement $\mathrm{du}$ polymère fondu avec des essais de microinjection. Ils montrent qu'une forte adhésion entre un polymère fondu et un substrat permet un meilleur remplissage des cavités par injection. Ils définissent un potentiel de démoulage $\Omega_{\mathrm{s}}$ tel que:

$$
\Omega_{S}=\int_{T_{n o}}^{T_{p}} S_{c} d T=\int_{T_{n o}}^{T_{p}} \gamma_{L}(\cos \theta-1) d T,
$$

où, $\theta$ est l'angle de contact entre le polymère fondu et le substrat, $\gamma_{L}$ est la tension superficielle du polymère fondu, $S_{\mathrm{c}}$ est le paramètre d'étalement, $T_{\mathrm{p}}$ est la température du polymère au contact du moule, $T_{\text {no }}$ est la température de non écoulement.
Ce potentiel représente l'intégrale du paramètre d'étalement entre la température d'injection et celle de figeage du polymère. Ils en déduisent qu'un potentiel faible doit impliquer une meilleure qualité de réplication, comme le montre la figure 10.

Cependant, une haute adhésion entre le substrat et la pièce injectée solidifiée peut entraîner une déformation, une détérioration de la pièce et/ou du moule $[57,83]$.

\subsection{Usinage des empreintes}

Dans la nano/microinjection, les empreintes de moules doivent servir à produire des pièces aux tolérances et dimensions submicroniques. Ainsi, des méthodes spécifiques doivent être utilisées pour réaliser les empreintes (Tab. 7). Nous ne présentons, dans cette section, que les technologies de lithographie et d'ablation laser à pulsions ultra-brèves, qui sont les plus documentées. D'autres techniques existent pour fabriquer des textures supérieures à la dizaine de micromètre tel que le grainage chimique pour lequel il est difficile de contrôler le motif. 


\subsubsection{Lithographie}

Le principe de la lithographie consiste à déposer une épaisseur donnée de résine sensible sur la surface du substrat à texturer, puis de la recouvrir d'un masque décrivant les motifs à réaliser. La surface est irradiée par un rayonnement qui, selon la nature de la résine, détruit ou durcit celle-ci sur toute son épaisseur. La résine restante est alors éliminée lors d'un bain dans un solvant adéquat. Les différents procédés de lithographie se différencient par le type de rayonnement utilisé : rayonnement UV, faisceaux de rayons X, d'ions ou d'électrons. Le choix de la source est directement lié à la résolution du motif qui peut varier de quelques nanomètres à quelques centaines de nanomètres [90-93]. Plus la longueur d'onde du rayonnement est faible, meilleure est la résolution. À titre d'exemple, la photolithographie permet d'atteindre des résolutions d'une centaine de nanomètres, tandis que la lithographie électronique offre des résolutions à l'échelle du nanomètre. Par cette méthode, on peut fabriquer des structures à très haut rapport d'aspect.

Initialement développées pour l'industrie électronique et ses matériaux (silicium, silice, or...), ces technologies ont pu être transposées aux moules métalliques par le dépôt, préalable au procédé de lithographie, d'une couche de nickel électrolytique plus ou moins dopé en fonction des caractéristiques mécaniques souhaitées, on parle alors du procédé LIGA (Lithographie, galvanoplastie, formage) [93]. Ainsi la zone texturée ou gravée est principalement le dépôt de nickel [83]. Des empreintes en silicium ont parfois été utilisées mais leur fragilité limite les domaines d'application [94]. Indépendamment du nombre limité de matériaux pouvant être gravés, cette technique a l'inconvénient majeur de nécessiter l'utilisation de produits chimiques dangereux, de salle blanche avec une durée de procédé importante. En revanche la précision de la technique permet d'obtenir les meilleures qualités dimensionnelles des motifs [87,93]. La figure 11 montre un insert texturé par cette technique et la pièce reproduite par injection.

\subsubsection{Texturation par laser}

Une autre méthode pour générer des motifs submicroniques sur la surface est l'utilisation d'un laser femtoseconde. Les mécanismes d'ablation avec le laser femtoseconde varient selon les matériaux et les conditions expérimentales. Ils se caractérisent par des puissances très élevées, de l'ordre de $10^{14} \mathrm{~W} . \mathrm{cm}^{-2}$ pendant des temps d'interaction lasermatière très courts ( $<$ picoseconde) qui, dans cette première phase, n'impliquent que les électrons de la cible et conduisent à qualifier le processus souvent d'athermique. Plusieurs explications ont été proposées telles que l'ablation non thermique [95], la transition solide-vapeur directe [96], l'explosion de phase [97,98] et l'explosion de Coulomb $[99,100]$. L'initiation et l'évolution de la formation du LIPSS est souvent concomitante au processus ablatif et conduit à la superposition de motifs.

En régime ablatif la résolution des motifs dépend du diamètre du faisceau laser, souvent de l'ordre de $25 \mu \mathrm{m}$ en fonction du système optique utilisé (lentilles...). Toutefois, Houzet et al. ont récemment montré qu'il était possible de
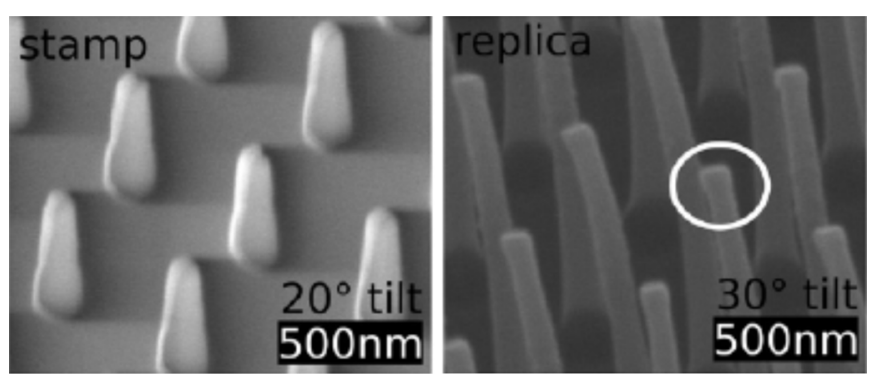

Fig. 11. Images MEB de l'empreinte réalisée par lithographie (stamp) et de la pièce en polycarbonate obtenue par injection d'après [83].

Fig 11. SEM images of the mold made by lithography (stamp) and of the polycarbonate part obtained by injection according to [83].

mettre en forme le faisceau laser, et ainsi diminuer la taille élémentaire des motifs, pour traiter des outillages d'injection et répliquer ces motifs sur les pièces plastiques avec succès (Fig. 12) [101].

L'un des avantages majeurs de la technique de gravure par laser est qu'elle permet l'usinage de tout type de matériaux, aussi bien les métaux que les céramiques, les verres et les polymères, si la longueur d'onde et la puissance du laser sont convenablement adaptées. Les nouvelles structures fabriquées au moyen du laser femtoseconde ont beaucoup attirées l'attention, au regard des différentes applications envisagées : codage optique [103], diminution du frottement élasto-hydrodynamique [104], de l'usure de polymère prothétique [105] mais aussi la texturation des outillages d'injection [106].

En effet, en régime femtoseconde, en fonction des conditions de traitement telles que la fluence du laser, le nombre d'impulsions, la polarisation et l'environnement d'irradiation, il est possible de produire de nouvelles micro/ nanostructures (avec des morphologies et des échelles caractéristiques différentes) [107,108]. Quelques exemples de ces textures sont présentées sur la figure 13.

On distingue principalement trois types de motifs induits par l'interaction laser matière dans ce régime:

- des ondulations submicroniques dont la période est de l'ordre de trois quarts la longueur d'onde du faisceau laser pour des amplitudes de quelques centaines de nanomètres (ou LIPPS, Laser Induced Periodic Surface Structures, parfois aussi appelées ripples);

- des ondulations dont la période est de quelques micromètres (LIPPS micrométriques) ;

- et les motifs générés par ablation de matière, $\Lambda_{1}$.

La formation de LIPPS a été reportée pour la première fois, en 1965, par Birnbaum [110] sur diverses surfaces de semi-conducteurs puis par d'autres chercheurs sur des surfaces lisses de cuivre, d'aluminium, de silicium ou de germanium [111-113]. Aujourd'hui, le mécanisme de formation des LIPPS est sujet à débat. Il peut résulter d'une ablation sélective du matériau par l'interférence entre le faisceau incident laser et la diffusion d'onde de surface [114] ou bien être le résultat d'une auto-organisation de la matière irradiée (à l'image de la formation des rides sur les dunes de sables) [115]. 


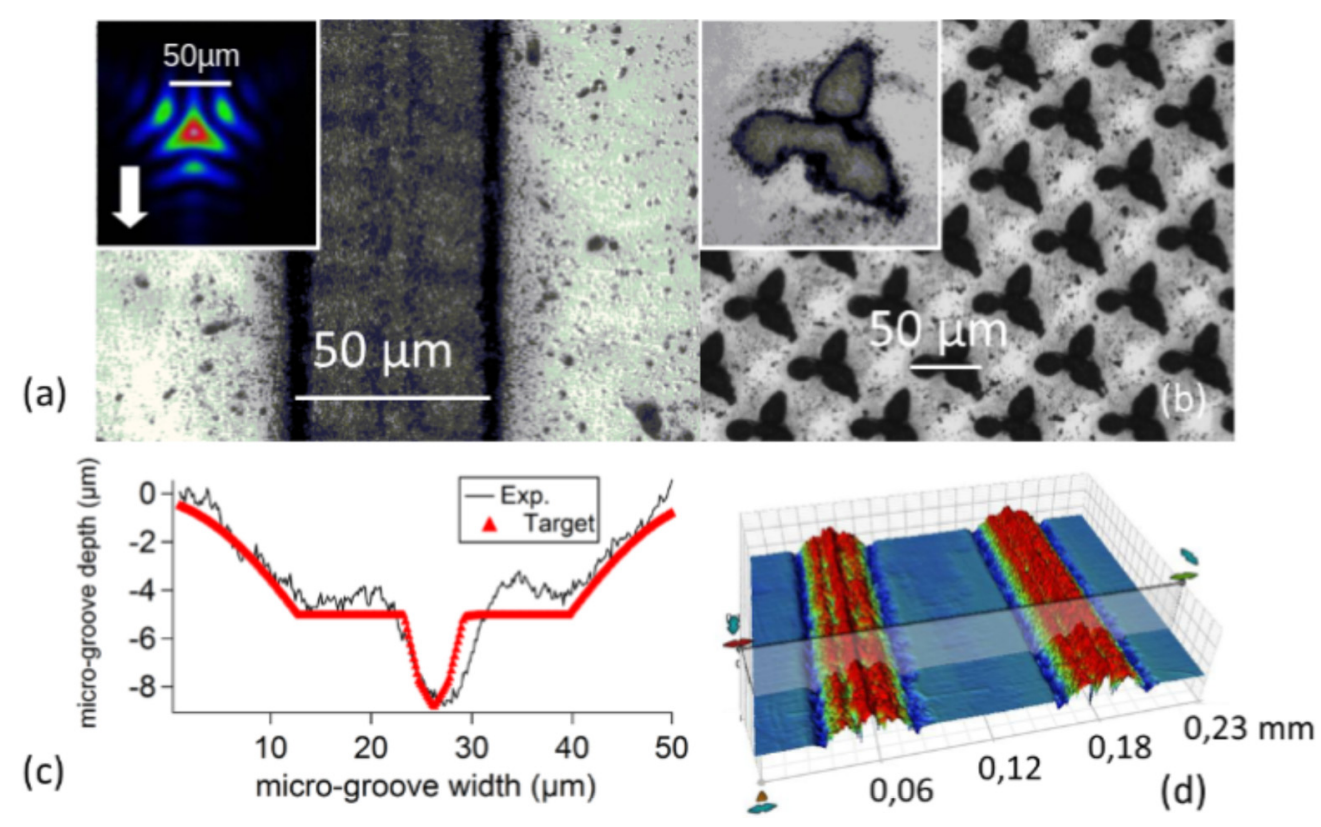

Fig. 12. (a) Micro-rainure (microscopie optique) ; (b) micro-bosses ; (c) profil horizontal et (d) image interférométrique d'une réplique injectée en PC de ce motif [101].

Fig 12. (a) Micro-groove (optical microscopy); (b) micro-bumps; (c) horizontal profile and (d) interferometric image of a PC-injected replica of this pattern [101].

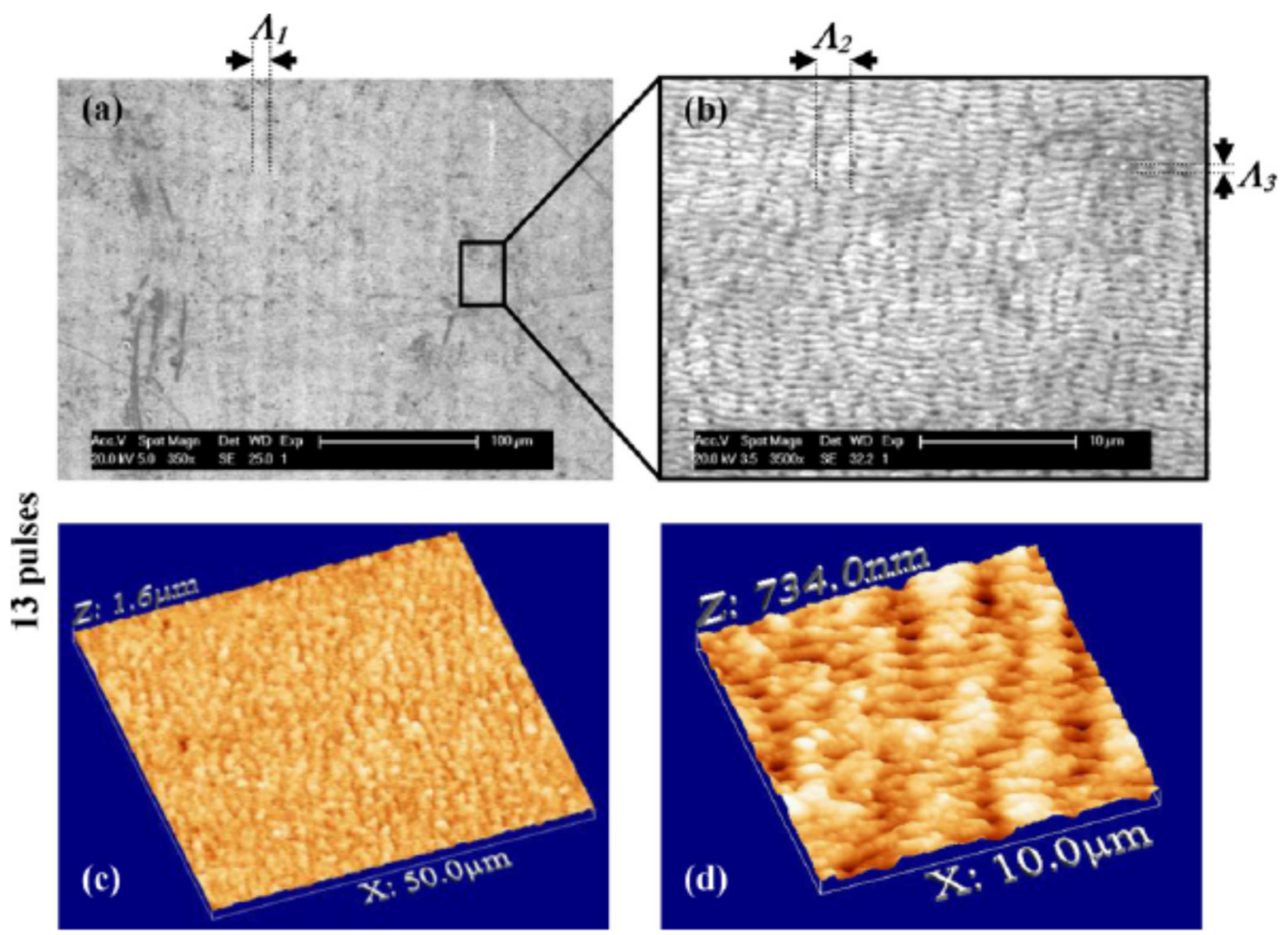

Fig. 13. (a et b) Images MEB ; (c et d) images AFM d'une texturation par laser femtoseconde d'un acier à moule [109]. Reproduit avec la permission de l'éditeur Elsevier.

Fig 13. ( $a$ and b) SEM images; (c and d) AFM images of femtosecond laser texturing of a mold steel [109]. 


\subsection{Quantification du taux de réplication}

Le procédé d'injection est par définition un procédé de reproduction où la pièce plastique est une reproduction de la texture présente à la surface du moule. En général, la reproduction n'est pas parfaite et la forme de la pièce injectée diffère du symétrique de la géométrie de l'empreinte. Il est donc particulièrement important de pouvoir contrôler cette réplique. On imagine aisément que la réplication d'une texture est d'autant plus difficile que le motif à répliquer est complexe et/ou à haut rapport de forme (rapport de la largeur sur sa hauteur). Pour des structures macroscopiques, les imperfections de reproduction sont souvent liées à des problèmes de retrait du polymère ou encore des apparitions de retassures sur la pièce injectée. Ces phénomènes sont assez bien connus et peuvent être prédit de manière analytique ou numérique avec une bonne précision $[116,117]$. En revanche, à des échelles submicroniques, la réplication est une question de transcription de la topographie de la surface du moule au polymère injecté $[39,118]$.

Dans la plupart des cas, lorsque les motifs sont relativement simples (trous, rainures...), pour exprimer la qualité de réplication, les auteurs s'attachent à comparer des dimensions verticales ou horizontales des motifs du moule et du réplica. Certains auteurs s'appuient sur des valeurs de hauteur, largeur ou de rapport de forme [119121] ou masse des pièces injectées [122] tandis que d'autres font des comparaisons visuelles [123]. Ces comparaisons sont satisfaisantes quand il s'agit de motifs très bien définis et périodiques comme ceux obtenus par LIGA, en revanche quand cela fait référence à des textures moins régulières et multi-échelles, cette approche locale ne convient plus. Il est indispensable de mettre en œuvre des outils statistiques susceptibles de décrire la topographie de surface au regard des caractéristiques de la forme souhaitée (paramètres d'anisotropie [38]), de la fonction envisagée [4] et de pouvoir faire des comparaisons quantitatives entre le moule et la pièce aux différentes échelles tels que présentés sur la figure 14, par exemple.

Pour ces structures, une autre approche a été utilisée regroupant des paramètres d'amplitude et de direction de structures [124,125]. La figure 14 présente la densité spectrale de puissance moyennée en fonction de la longueur d'onde déterminée à partir de mesures topographiques de surface de pièces en polypropylène et du moule correspondant [38]. L'analyse de ce type de courbes permet d'identifier une longueur d'onde principale des textures $(\approx 650 \mathrm{~nm})$ en adéquation avec celle du moule (à quelques dizaines de nanomètres près) ainsi que la puissance correspondante à cette longueur d'onde dont le rapport (pièce-moule) permet aussi de quantifier la qualité de cette réplication.

\subsection{Résumé}

En résumé l'efficience des revêtements en injection est souvent estimée soit globalement à partir d'une comparaison de la qualité des pièces entre celles issues d'outillages revêtus ou non, soit au regard d'une fonction très précise (tenue à l'usure abrasive ou adhésion moule-polymère),

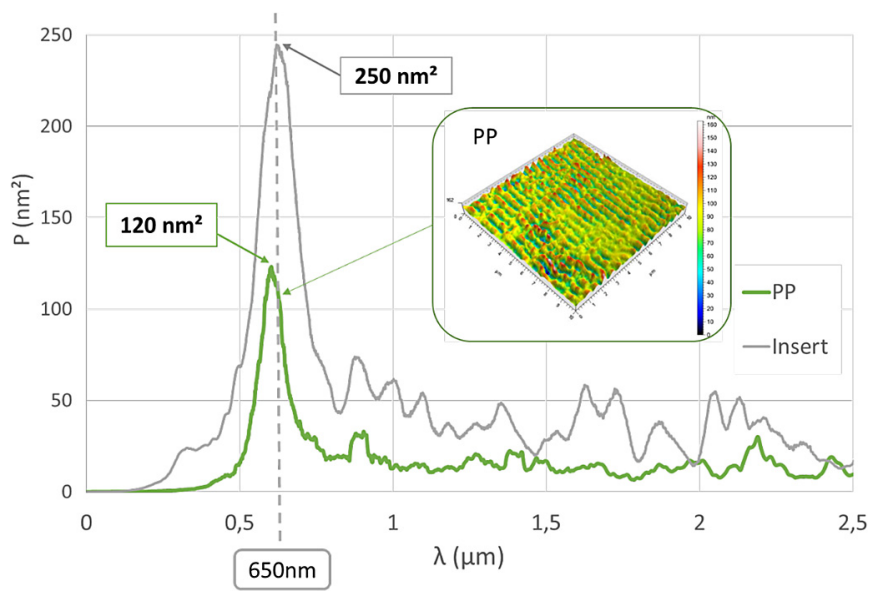

Fig. 14. Analyse spectrale d'un insert et d'une pièce injectée texturée en polypropylène $(\mathrm{PP})$ à partir de cet insert: densité spectrale de puissance moyennée $(\mathrm{P})$ en fonction de la longueur d'onde $(\lambda)$. Image $3 \mathrm{D}$ obtenue par interférométrie optique correspondant à la zone texturée de la pièce en $\mathrm{PP}$.

Fig 14. Spectral analysis of an insert and a textured injected piece of polypropylene $(P P)$ from this insert: mean power spectral density $(P)$ as a function of the wavelength $(\lambda)$. And the $3 D$ image obtained by optical interferometry corresponding to the textured area of PP part.

quantifiée ex situ dans des conditions plus ou moins représentatives des phénomènes réels se produisant durant l'injection.

Dans le premier cas l'information résultante est très pratique mais limite les interprétations physiques que l'on peut déduire des résultats, dans le deuxième cas, elle n'offre qu'une réponse fragmentaire qui peut occulter les phénomènes physiques en jeu. Ainsi l'influence du revêtement sur le procédé doit donc être défini à partir d'outils métrologiques quantitatifs et d'une approche globale du processus en tenant compte de ces multiples aspects (y compris les échanges thermiques moule-polymère) ainsi que les couplages entre eux.

\section{Procédé d'injection}

Le procédé d'injection se déroule en plusieurs étapes: le dosage, l'injection, le compactage (ou maintien), le refroidissement et la phase d'éjection. Parmi ces différentes phases nous avons recensé les paramètres du procédé qui influent tout particulièrement sur la qualité de réplication des pièces nano-structurées. Il s'agit de:

- la température du moule $\left(T_{\mathrm{m}}\right)$;

- la température du fourreau $\left(T_{\mathrm{fo}}\right)$ qui correspond à peu près à la température du polymère fondu;

- la vitesse d'injection $\left(V_{\mathrm{i}}\right)$;

- la pression $\left(P_{\mathrm{m}}\right)$ et le temps de maintien.

\subsection{Impact des paramètres d'injection sur la réplication}

À partir des travaux de différents auteurs, le tableau 8 recense de manière non exhaustive l'impact des paramètres 
Tableau 8. Conditions d'injection améliorant ou non la réplication de surfaces submicroniques. $V_{\mathrm{i}}$ : vitesse d'injection; $T_{\mathrm{f}}$ : température fourreau; $T_{\mathrm{m}}$ : température moule; $P_{\mathrm{m}}$ : Pression de maintien; $h_{\mathrm{R}}$ : hauteur/profondeur du motif; $w_{\mathrm{R}}$ : largeur du motif ; $e$ : épaisseur de la pièce. Les effets les plus importants sont notés «+»et «NC» correspond à des grandeurs non mesurées.

Table 8. Injection conditions improving, or not, the replication of submicron surfaces. $V_{i}$ : Injection speed; $T_{f}:$ melt temperature; $T_{m}$ : mold temperature; $P_{m}$ : Holding pressure; $h_{R}$ : height / depth of pattern; $w_{R}$ : pattern width; e: Thickness of the part. The most important effects are marked "+" and "NC" corresponds to unmeasured variables.

\begin{tabular}{|c|c|c|c|c|c|c|c|c|}
\hline \multirow[t]{2}{*}{$\begin{array}{l}\text { Taille des } \\
\text { structures }\end{array}$} & \multirow[t]{2}{*}{$\begin{array}{l}\text { Forme des } \\
\text { textures }\end{array}$} & \multirow[t]{2}{*}{ Polymère } & \multicolumn{4}{|c|}{$\begin{array}{l}\text { Paramètres procédés pour une } \\
\text { meilleure réplication }\end{array}$} & \multirow[t]{2}{*}{$\begin{array}{l}\% \text { Réplication ou } \\
\text { remarques majeures }\end{array}$} & \multirow[t]{2}{*}{ Références } \\
\hline & & & $\begin{array}{l}V_{\mathrm{i}} \\
\left(\mathrm{cm}^{3} \cdot \mathrm{s}^{-1}\right)\end{array}$ & $T_{\mathrm{fo}}$ & $T_{\mathrm{m}}$ & $P_{\mathrm{m}}$ & & \\
\hline $\begin{array}{l}\varnothing=300 \mathrm{~nm} \\
\lambda=600 \mathrm{~nm} \\
h_{\mathrm{R}}=600 \mathrm{~nm}\end{array}$ & Trous & $\begin{array}{l}\text { PMMA } \\
\text { A-PA }\end{array}$ & $\begin{array}{l}15 \\
15\end{array}$ & $\begin{array}{l}250 \\
280\end{array}$ & $\begin{array}{l}80 \\
70\end{array}$ & $\begin{array}{l}800 \\
800\end{array}$ & $\begin{array}{l}25 \% \\
50 \%\end{array}$ & {$[126]$} \\
\hline Quelques mg & $\mu$ pièce & $\begin{array}{l}\text { POM } \\
\text { LCP }\end{array}$ & $\begin{array}{l}0 \\
+\end{array}$ & $\begin{array}{l}+ \\
0\end{array}$ & - & $\begin{array}{l}++ \\
++\end{array}$ & $\begin{array}{l}\text { Masse et dimensions des } \\
\text { pièces }\end{array}$ & {$[127]$} \\
\hline $\begin{array}{l}h_{\mathrm{R}}=110 \mathrm{~nm} \\
w_{\mathrm{R}}=40 \mathrm{~nm}\end{array}$ & Piliers & $\mathrm{COC}$ & & & + & & $\begin{array}{l}\text { Quel que soit la chimie } \\
\text { du moule }\end{array}$ & {$[72]$} \\
\hline $\begin{array}{l}h_{\mathrm{R}}=0,66-2,0 \mu \mathrm{m} \\
w_{\mathrm{R}}=10-150 \mathrm{~nm}\end{array}$ & Piliers & $\mathrm{PC}$ & $\mathrm{NC}$ & + & & $\mathrm{NC}$ & $\begin{array}{l}\text { Jusqu'à } 95 \% \text { de } \\
\text { répliques }(\text { avec } \\
\left.\text { revêtement } \mathrm{SiO}_{2}\right)\end{array}$ & {$[83]$} \\
\hline $\begin{array}{l}h_{\mathrm{R}}=35-105 \mathrm{~nm} \\
w_{\mathrm{R}}=50-100 \mathrm{~nm}\end{array}$ & Piliers & $\mathrm{COC}$ & $<4 \mathrm{~cm}^{3} / \mathrm{s}$ & $\mathrm{NC}$ & $\mathrm{NC}$ & + & $\begin{array}{l}\text { Attention aux } \\
\text { contraintes internes en } \\
\text { appliquant beaucoup de } \\
\text { maintien }\end{array}$ & [128] \\
\hline $\begin{array}{l}h_{\mathrm{R}}=530 \mathrm{~nm} \\
w_{\mathrm{R}}=600 \mathrm{~nm}\end{array}$ & Canaux & $\begin{array}{l}\text { PC } \\
\text { PMMA }\end{array}$ & + & + & + & + & $\begin{array}{l}\text { Jusqu'à } 95 \% \text { en milieu } \\
\text { et fin de pièce }\end{array}$ & {$[47]$} \\
\hline$e=30 \mu \mathrm{m}$ & Micro-pièce & $\begin{array}{l}\text { POM } \\
\text { HDPE } \\
\text { PC }\end{array}$ & $\begin{array}{l}+ \\
+ \\
+\end{array}$ & & & & & [129] \\
\hline $\begin{array}{l}h_{\mathrm{R}}=670 \mathrm{~nm} \\
w_{\mathrm{R}}=0,5-1 \mu \mathrm{m}\end{array}$ & Canaux & $\begin{array}{l}\text { PC } \\
\text { COP } \\
\text { SAN } \\
\text { HFP-TFE-Et }\end{array}$ & $\begin{array}{l}+ \\
+ \\
+ \\
+\end{array}$ & & & & $\begin{array}{l}\mathrm{PC}>\mathrm{SAN} \text { et les } \\
\text { répliques sont meilleures } \\
\text { avec le motif où } \\
w_{\mathrm{R}}=1 \mu \mathrm{m} \text { sauf pour le } \\
\mathrm{SC} \text { qui a la même } \\
\text { hauteur de réplique quel } \\
\text { que soit la hauteur du } \\
\text { réseau }\end{array}$ & {$[121]$} \\
\hline $\begin{array}{l}\text { Textures } \\
\min =150 \mathrm{~nm}\end{array}$ & $\begin{array}{l}\text { Logo } \\
\ll U C D »\end{array}$ & PEHD & & & ++ & + & $\begin{array}{l}\text { Qualité dépend de la } \\
\text { direction des textures } \\
\text { vs. direction du flux } \\
\text { d'injection }\end{array}$ & [119] \\
\hline
\end{tabular}

d'injection sur la qualité des répliques de pièces submicroniques en fonction des polymères et des textures.

On observe d'après ce tableau qu'il n'y a pas un ou deux facteurs identiques qui ressortent comme étant les paramètres les plus importants dans la réplication par injection de textures submicroniques. En effet, la vitesse d'injection $\left(V_{\mathrm{inj}}\right)$ semble être le facteur prédominant dans plusieurs études [121,129] alors que dans une autre [47] tous les facteurs semblent influencer de la même manière la qualité de réplique, malgré qu'ils utilisent le même polymère (un PC).
Dans ce tableau, seules les mesures de qualité de répliques par topographie (AFM, MEB...) sont prises en compte et les travaux de Miikkulainen [130] en sont absents car la qualité de la réplique est mesurée par des essais d'angle de contact. Il s'agit, dans cette étude, de poser une goutte d'eau sur la surface texturée et de comparer l'angle que fait cette goutte avec la surface. Sur du PP, la différence d'angle de contact pour un même diamètre de pore n'est pas significative pour les trois températures de moule, tandis que leur hauteur varie entre 20 et $100 \mathrm{~nm}$ (Fig. 15). Ainsi, dans cette étude, la température du moule 


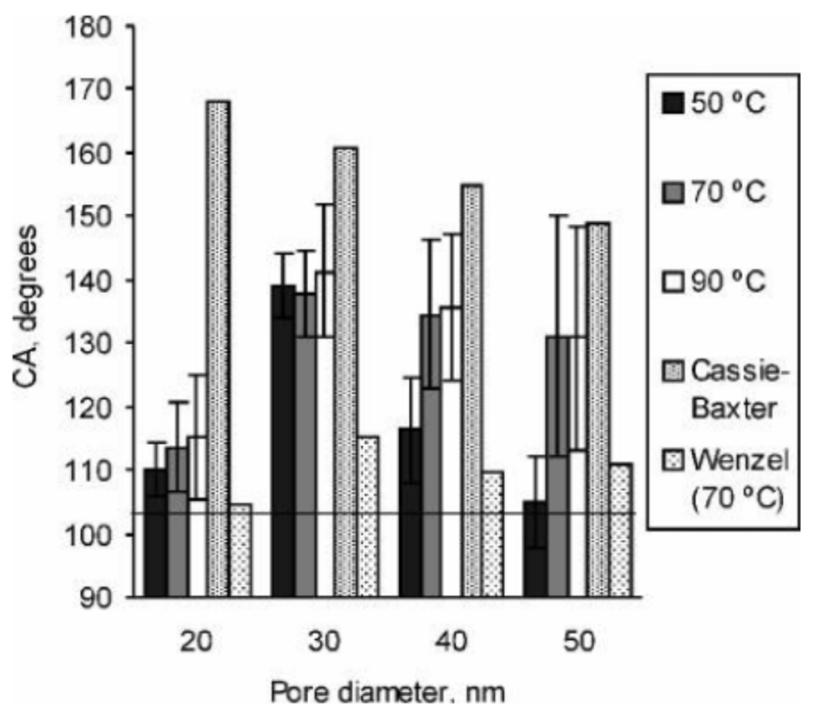

Fig. 15. Angles de contact sur une pièce en PP en fonction de la taille des pores. Les pièces ont été injectées à différentes températures de moule $\left(50,70\right.$ et $\left.90^{\circ} \mathrm{C}\right)$. La ligne horizontale correspond à l'angle de contact du PP sur surface lisse, d'après [130]. Reproduit avec la permission de American Chemical Society.

Fig 15. Contact angles on a PP part according to the size of the pores. The pieces were injected at different mold temperatures $\left(50,70\right.$ and $\left.90^{\circ} \mathrm{C}\right)$. The horizontal line corresponds to the contact angle of the PP on smooth surface, according to [130].

ne semble pas être un facteur prédominant dans la reproduction de ces pores.

\subsection{Position de la texture}

La position sur la pièce de la texture à répliquer influence aussi la réplication. Ito et al. [47] observent qu'avec du PC, la qualité de réplique est meilleure en fin de pièce (en fonction du sens d'écoulement du polymère dans l'empreinte) (95\%) qu'en début (20\%) (Fig. 16). Selon eux, cette différence n'est pas due à la distribution de pression dans la cavité, minimale en fin de pièce, là où la réplique est la meilleure. À partir de mesures optiques en lumière polarisée sur les pièces injectées, ils montrent que celles-ci présentent des contraintes résiduelles importantes en début de pièce (zone de pression maximale) et ils leur attribuent l'origine des déformations de la pièce lors de la phase d'éjection.

De la même manière, Sha et al. [123] mettent en évidence l'importance de la position des textures par rapport au seuil d'injection. De plus, ils observent que la distance entre les différentes microstructures n'affecte pas leur remplissage. Les trois polymères utilisés pour cette étude (PP, POM et ABS) ne montrent aucune différence dans leur qualité de réplique suivant la distance qui sépare les micro-trous ou les micro-cavités. Ainsi, ils en concluent que le flux de polymère fondu n'est pas sensible à cette distance. Les principaux facteurs à prendre en compte pour améliorer la réplique de nanostructures sont les paramètres procédés, la taille des textures et la position des textures par rapport au seuil d'injection.

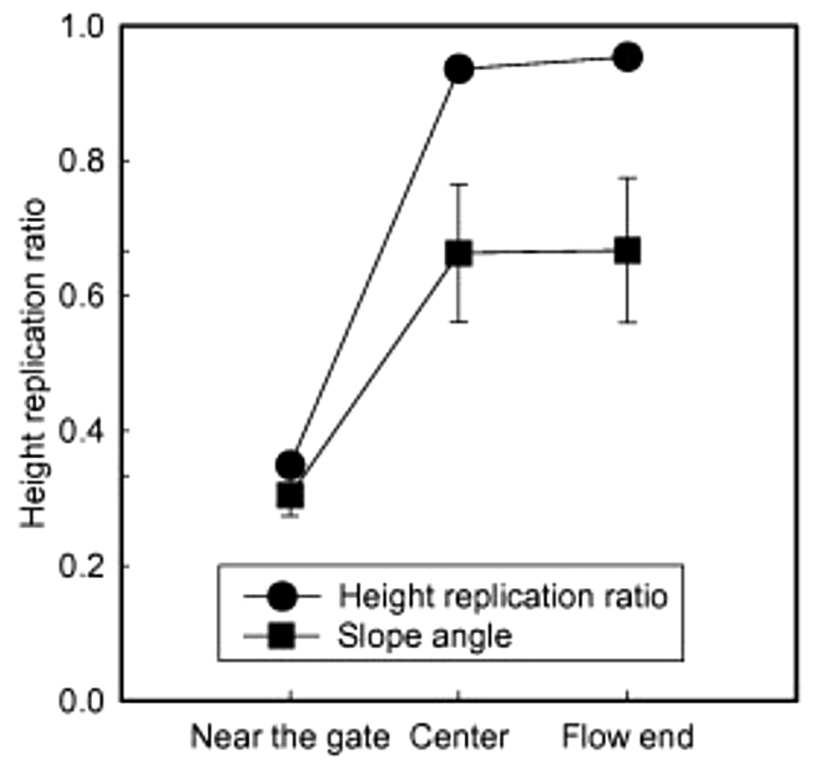

Fig. 16. Hauteur des répliques en fonction de la position des textures, d'après [47]. Reproduit avec la permission de l'éditeur Elsevier.

Fig 16. Height of the replicas according to the position of the textures, according to [47].

\subsection{Remplissage des cavités}

En microinjection il est particulièrement important de tenir compte des conditions de remplissage des cavités lors de l'injection. Lors de ce processus, le polymère fondu subit des contraintes qui impactent le remplissage de la cavité du moule et donc la qualité de la pièce finale. Ainsi, il est possible d'estimer le taux de cisaillement $(\dot{\gamma})$ et la contrainte imposée $(\sigma)$, dans les hypothèses d'un écoulement laminaire à régime permanent sans glissement à la paroi (vitesse tangentielle à la surface du solide est nulle) dans une plaque, [131] tels que:

$$
\dot{\gamma}=\frac{6 Q}{w e^{2}},(\mathrm{~V}-1) \text { et } \sigma=\frac{\mathrm{we}}{2(\mathrm{w}+\mathrm{h})}\left(\frac{-\triangle \mathrm{P}}{\mathrm{L}}\right),(\mathrm{V}-2)
$$

avec, $L$ : longueur de la pièce, $w$ : largeur de la pièce, $e$ : épaisseur de la pièce, $Q$ : débit, $\Delta P$ : correspond à la chute de pression.

À partir de ces équations, Chien et al. [40] proposent un modèle rhéologique pour modéliser le remplissage des micropièces. Il est admis que la viscosité d'un polymère fondu à travers une micro-pièce ou un micro-canal est plus faible qu'à travers un canal de taille standard dans lequel le glissement à la paroi joue un rôle prédominant. Ainsi, ils décomposent le débit total à travers le micro-canal (ou micro-pièce) $Q_{\text {tot }}$ comme la somme du débit avec glissement $\left(Q_{\text {slip }}\right)$ et du débit sans glissement $\left(Q_{\text {no-slip }}\right)$ tel que :

$$
Q_{t o t}=Q_{n o-s l i p}+Q_{\text {slip }} \text {. }
$$

Ils comparent leurs mesures de cisaillement dans un micro-sillon par rapport à une mesure conventionnelle de rhéologie capillaire et observent que plus la taille des microcanaux est faible, plus le polymère est visqueux (voir Fig. 17). 


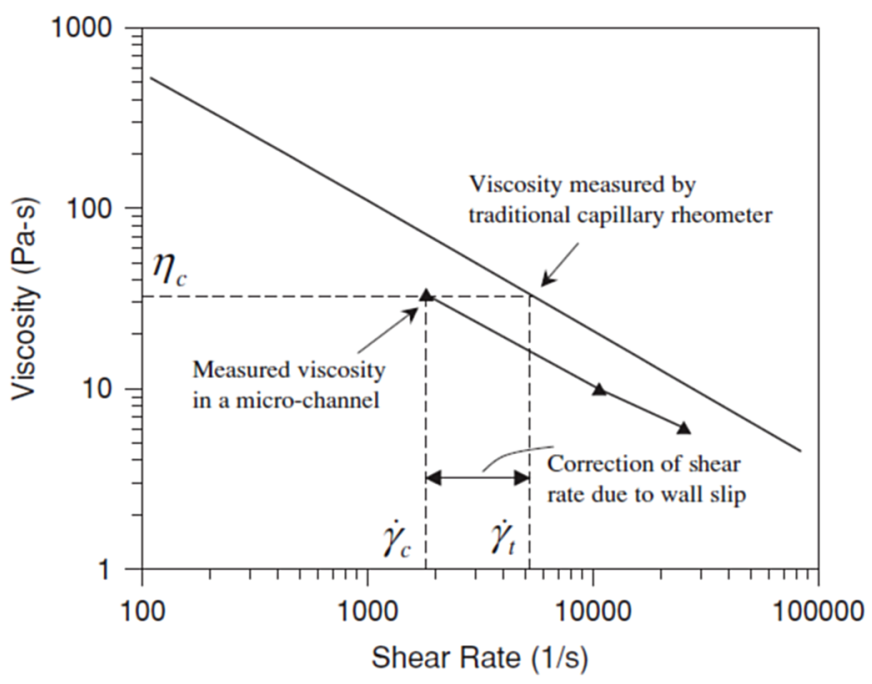

Fig. 17. Viscosité en fonction du taux de cisaillement avec $\dot{\gamma}_{c}$, la viscosité du polymère dans le microsillon, et $\dot{\gamma}_{t}$, la viscosité du polymère dans un rhéomètre capillaire pour une même viscosité, d'après [40]. Reproduit avec la permission de l'éditeur IOPscience.

Fig 17. Viscosity as a function of the shear rate with $\dot{\gamma}_{c}$, the viscosity of the polymer in the micro-groove, and $\dot{\gamma}_{t}$, the viscosity of the polymer in a capillary rheometer for the same viscosity, according to [40].

À partir de cette figure, ils déduisent le débit avec glissement à la paroi $\left(Q_{\text {slip }}\right)$ tel que :

$$
Q_{\text {slip }}=\frac{\pi R^{3}}{4\left(\frac{3}{4}+\frac{1}{4} b\right)}\left(\dot{\gamma}_{t}-\dot{\gamma}_{c}\right)
$$

avec, $R$ : rayon du canal, $b$ : pente de $\log \dot{\gamma}$ en fonction de log $\delta$ (similaire à la correction de Rabinowitch), $\dot{\gamma}_{\mathrm{c}}$ : viscosité du polymère dans le microsillon, $\dot{\gamma}_{t}$ : viscosité du polymère dans un rhéomètre capillaire

On observe que l'écoulement à la paroi est la cause de la réduction de viscosité dans le micro-canal. L'étude indique qu'une modélisation appropriée du remplissage des cavités lors de la microinjection passe par une meilleure connaissance des paramètres rhéologiques des polymères, permettant ainsi une modélisation précise des phénomènes présents lors de la microinjection.

\subsection{Cinétique de solidification}

Dans un procédé classique d'injection, le temps de refroidissement du polymère est souvent estimé par $[85,132]$ :

$$
t_{r}=\frac{s}{\alpha \pi} \times \ln \left(\frac{\pi}{\pi} \times \frac{T_{M}-T_{m}}{T_{\mathrm{e}}-T_{m}}\right),
$$

avec, $t_{\mathrm{r}}$ : temps de refroidissement, $S$ : épaisseur maximum de la cavité, $T_{\mathrm{M}}$ : température de la matière, $T_{\mathrm{m}}$ : température moyenne du moule, $\alpha$ : diffusivité thermique.

Cependant, pour des pièces avec une géométrie complexe, une modélisation différente est nécessaire. Ainsi, le rapide refroidissement du polymère au contact de l'empreinte est responsable des «effets de peau » présents sur la pièce injectée (voir Sect. 2). Pour un problème de reproduction de textures, la cinétique d'imprégnation des textures du moule par le polymère est à mettre en parallèle de la cinétique de formation de la gaine solide.

\subsection{Estimation de la taille de gaine solide}

La qualité des répliques de petites structures (échelle du nanomètre ou du micromètre) est intimement liée à la formation d'une gaine solide lors du contact du polymère chaud sur les parois du moule froid. Cette solidification pourrait empêcher le remplissage de ces motifs si cette gaine solide est trop épaisse [126]. La détermination expérimentale de l'épaisseur de cette gaine en fonction du temps est difficile à mesurer. Berge et al. [133] proposent de déterminer cette épaisseur suivant l'équation ci-dessous :

$$
\delta(t)=1,9 \times \frac{T_{n o}-T_{m}}{T_{f}-T_{m}} \times \sqrt{\frac{\alpha_{p} \times t_{c}}{\rho_{m} \times c_{p}}} .
$$

Sa croissance est rapide, en effet, elle peut atteindre plusieurs dizaines de micromètres en quelques dixièmes de seconde $[60,74,126]$. Cette vitesse dépend de la température à l'interface moule/polymère mais cette température est différente de la température interne du moule. En effet, $T_{\mathrm{m}}$ est instantanément augmentée au contact du polymère fondu encore chaud. L'évolution de cette température instantanée $\left(T_{\mathrm{mi}}\right)$ a été calculée et s'exprime sous la forme $[134,135]:$

$$
\frac{T_{f}-T_{p}}{T_{p}-T_{m i}}=\frac{(K \rho c)_{m}}{(K \rho c)_{p}}
$$

avec, l'indice « $m$ » correspondant aux valeurs relatives au moule et l'indice « $p »$ pour celles du polymère, $K$ : conductivité thermique, $\rho$ : densité, $c$ : chaleur spécifique.

Ainsi, pour augmenter la température à l'interface, il faut une faible valeur de $K, \rho$ et/ou $c$. Pour ralentir la croissance de la gaine solide, l'utilisation de revêtements avec une faible conductivité thermique peut être préconisée. Elle permettrait une meilleure qualité de reproduction des structures comme l'a montré Luchetta et al. [136]. Cette problématique est assez peu étudiée alors qu'elle semble être un des points clé dans le procédé de microinjection et plus particulièrement lors de la réplication de nano-structure par injection.

\section{Conclusion}

La microinjection est un procédé complexe à mettre en œuvre qui permet de produire des surfaces texturées en grandes séries à moindre coût. Dans cet article, différents aspects de la réplication de textures submicroniques ont été abordés tels que les matériaux utilisés (polymère injecté et surface de l'empreinte) et les conditions d'injection. Comparé au procédé d'injection traditionnel, le procédé de microinjection implique une évolution technologique 
significative. En effet, la microinjection ne consiste pas seulement en une diminution de taille par rapport au procédé d'injection classique.

La complexité du procédé de microinjection vient d'abord du choix des matériaux. En effet, la structure des polymères a une importance notable. La connaissance des différentes propriétés thermiques $\left(\mathrm{T}_{\mathrm{g}}, \mathrm{T}_{\mathrm{f}} \ldots\right)$ et rhéologiques est essentielle dans le but d'une production de pièces de qualité. Les caractéristiques du moule, notamment la chimie de surface des parties moulantes et de leurs motifs impactent la qualité de la pièce finale obtenue. En effet, lors du remplissage de l'empreinte par le polymère fondu, il faut, à la fois, avoir une bonne imprégnation des microcavités par ce polymère (souvent synonyme d'une bonne adhésion polymère fondu/moule), et d'autre part, une faible adhérence entre le polymère solidifié et le moule afin d'éviter tout problème de «collage » et de déformation lors de l'éjection de la pièce. Les fonctions de bonne imprégnation polymère fondu/moule et de faible adhérence polymère solidifié/moule peuvent être obtenues par un dépôt d'un film mince sur la surface de l'empreinte. En effet, différentes études ont montré que des revêtements à base de nitrures métalliques ou bien de DLC (Diamond Like Carbon) peuvent améliorer la durée de vie du moule, l'adhésion entre le polymère fondu et le moule, ou encore diminuer la friction lors de l'éjection de la pièce. En revanche, très peu de travaux prennent en considération l'influence du revêtement sur la thermique du contact. Ceci peut s'expliquer par le fait que la caractérisation des propriétés thermiques du moule nécessite des mesures de microscopiques difficiles à mettre en œuvre.

Selon les différents paramètres intervenant lors du procédé de microinjection, les conditions d'injection sont aussi d'une grande importance et diffèrent du procédé d'injection classique. Selon Giboz et al. [85], la température du moule et la pression d'injection doivent être augmentées dans le cas de la microinjection, ce qui aboutit à une augmentation significative du temps de cycle. À ce sujet, Yao et al. [134] expliquent que la formation et la croissance de la gaine solide durant la phase d'injection est responsable de la qualité de la pièce finale. Ainsi, une solution serait de chauffer rapidement le moule avant l'injection pour le refroidir rapidement après injection. Cependant cette technologie de chauffe et de refroidissement rapide n'est pas encore très développée, voire quasiment inexistante, en industrie. Dans la plupart des cas, les auteurs s'accordent pour dire que les températures de la matière et $\mathrm{du}$ moule, la vitesse d'injection et le maintien impactent la qualité de réplication des motifs de la pièce obtenue. Cependant, l'importance relative de ces paramètres sur la qualité des textures obtenues diffère souvent d'un auteur à l'autre.

\section{Nomenclature}

a Paramètre sans dimension décrivant la transition entre le premier plateau newtonien et la loi puissance

A Surface de contact de la pièce sur le noyau

ABS Acrylonitrile Butadiène Styrène
AFM Microscope à Force Atomique

$b \quad$ Pente de $\log$ en fonction de $\log \delta$ (similaire à la correction de Rabinowitch)

c Chaleur spécifique

COC Copolymère cyclo-oléfine

CrN Nitrure de Chrome

CVD Chemical Vapor Deposition

$D \quad$ Diamètre du noyau

DLC Diamond Like Carbon

$e \quad$ Épaisseur

$F_{\mathrm{a}} \quad$ Force adhésive

$F_{\mathrm{c}} \quad$ Force de cisaillement

$F_{\text {é }} \quad$ Force nécessaire à l'éjection de la pièce

FOTS Revêtement fluoroctatrichlorosilane

FS Revêtement fluorosilane

$\mathrm{h}_{\mathrm{r}, \mathrm{R}} \quad$ Hauteur de réplique (sur la pièce), $r$, du motif initial (sur le moule) R

HSQ Revêtement Hydrogène Sisesquioxane

indice $m$ Concerne le moule

indice $p \quad$ Concerne le polymère

IP Indice de polymolécularité

$k \quad$ Déterminé à partir du module de Young

$L \quad$ Longueur de la pièce ou du capillaire

LIGA Lithographie

$m \quad$ Coefficient de la loi puissance

MEB Microscope Électronique à Balayage

MFI «Melt Flow Index » (viscosité à faible cisaillement)

$M_{\mathrm{n}} \quad$ Masse molaire moyenne en nombre

MoN Revêtement nitrure de molybdène

$m_{\mathrm{p}} \quad$ Masse du polymère

$M_{\mathrm{w}} \quad$ Masse molaire moyenne en masse

$M_{\mathrm{z}} \quad$ Taille moyenne

$v \quad$ Coefficient de poisson du polymère

$\eta \quad$ Viscosité

$\eta_{0} \quad$ Viscosité à cisaillement nul

$P \quad$ Densité spectrale de puissance moyennée

PA Polyamide

PACVD Plasma Assisted Chemical Vapor Deposition

PBT Polybutadiène Téréphtalate

PC Polycarbonate

$\mathrm{Pc} \quad$ Pression commutation

PEHD Polyéthylène Haute Densité

PET Polyéthylène Téréphtalate

PI Indice de polydispersité

Pm Pression de maintien

PMMA Polyméthacrylate de méthyl

PMP Polyméthylpentène

POM Polyoxyméthylène

PP Polypropylène

PS Polystyrène

PVC Polychlorure de Vinyle

PVD Physical Vapor Deposition

$Q \quad$ Débit

$Q_{\text {no-slip } \quad \text { Débit avec glissement }}$

$Q_{\text {slip }} \quad$ Débit sans glissement

$Q_{\text {tot }} \quad$ Débit total

$R c \quad$ Rayon effectif de l'interface ou rayon du canal

$R \quad$ Taux de réplication

$R_{\mathrm{c}} \quad$ Rayon du capillaire

$S \quad$ Épaisseur maximale 
$S_{\mathrm{a}} \quad$ Moyenne arithmétique de la surface

$S_{\mathrm{c}} \quad$ Paramètre d'étalement

SFE Énergie de surface

SiOC Revêtement organosilicone

$T \quad$ Température

$t_{\mathrm{c}} \quad$ Temps de contact

$T_{\text {é }} \quad$ Température d'éjection

$T_{\mathrm{f}} \quad$ Température de fusion

$T_{\mathrm{fo}} \quad$ Température du fourreau

$\mathrm{T}_{\mathrm{g}} \quad$ Température de transition vitreuse

TiN Nitrure de titane

$T_{\mathrm{m}} \quad$ Température du moule

$T_{\mathrm{M}} \quad$ Température de la matière

$T_{\text {no }} \quad$ Température de non écoulement du polymère

$T_{\mathrm{p}} \quad$ Température à l'interface moule/polymère fondu

TPU Polyuréthane thermoplastique

$t_{\mathrm{r}} \quad$ Temps de refroidissement

$V \quad$ Vitesse

$V_{\mathrm{i}} \quad$ Vitesse d'injection

$w \quad$ Largeur de la pièce

$W_{\text {ab }} \quad$ Travail d'adhésion entre un solide $a$ et un solide $b$

WN Revêtement de nitrure de Tungstène

$\dot{\gamma} \quad$ Taux de cisaillement

$\dot{\gamma}_{c} \quad$ Viscosité du polymère dans le microsillon ou un rhéomètre capillaire

$\alpha \quad$ Coefficient de dilatation thermique du polymère

$\delta \quad$ Épaisseur de gaine solide

$\Delta P \quad$ Chute de pression

$\Delta T \quad$ Différence entre la température de ramollissement du polymère et la température du moule

$\theta \quad$ Angle de contact

$\lambda \quad$ Longueur d'onde

$\mu \quad$ Coefficient de friction

$\rho \quad$ Densité

$\sigma \quad$ Contrainte imposée

$\gamma_{L} \quad$ Tension superficielle du polymère fondu

$\gamma_{s} \quad$ Tension de surface

$\gamma_{s}^{d} \quad$ Tension de surface dispersive

$\gamma_{s L} \quad$ Tension de surface de l'interface solide-liquide

$\gamma_{s} \quad$ Tension de surface polaire

$\Omega_{\mathrm{S}} \quad$ Potentiel de démoulage

\section{References}

1. «Publication du Panorama 2015: découvrez les indicateurs économiques de référence de la plasturgie et des composites - Fédération Plasturgie et Composites. Fédération de la plasturgie et des composites

2. S.G. Harris, E.D. Doyle, Y.-C. Wong, P.R. Munroe, J.M. Cairney, J.M. Long, Reducing the macroparticle content of cathodic arc evaporated TiN coatings, Surf. Coat. Technol. 183(2-3), 283 (2004)

3. B. Bhushan, H. Fuchs, M. Tomitori, Applied scanning probe methods $\mathrm{X}$ : biomimetics and industrial applications, Springer-Verlag, Berlin Heidelberg, Vol. 9, 2008

4. V. Belaud, S. Valette, G. Stremsdoerfer, M. Bigerelle, S. Benayoun, Wettability versus roughness: Multi-scales approach, Tribol. Int. 82B, 343 (2015)
5. M. Nosonovsky, B. Bhushan, Multiscale Dissipative Mechanisms and Hierarchical Surfaces: Friction, Superhydrophobicity, and Biomimetics, Springer Science \& Business Media, 2008

6. B. Bhushan, K. Koch, Y.C. Jung, Fabrication and characterization of the hierarchical structure for superhydrophobicity and self-cleaning, Ultramicroscopy 109(8), 1029 (2009)

7. D. Kontziampasis, G. Boulousis, A. Smyrnakis, K. Ellinas, A. Tserepi, E. Gogolides, Biomimetic, antireflective, superhydrophobic and oleophobic PMMA and PMMA-coated glass surfaces fabricated by plasma processing, Microelectron. Eng. 121, 33 (2014)

8. Y. Li, et al., Biomimetic Surfaces for High-Performance Optics, Adv. Mater. 21(46), 4731 (2009)

9. P. Comanns, C. Effertz, F. Hischen, K. Staudt, W. Böhme, W. Baumgartner, Moisture harvesting and water transport through specialized micro-structures on the integument of lizards, Beilstein J. Nanotechnol. 2(1), 204 (2011)

10. S. Niu, et al., Excellent Structure-Based Multifunction of Morpho Butterfly Wings: A Review, J. Bionic Eng. 12(2), 170 (2015)

11. B.R. Whiteside, M.T. Martyn, P.D. Coates, P.S. Allan, P.R. Hornsby, G. Greenway, Micromoulding: process characteristics and product properties, Plast. Rubber Compos. 32 (6), 231 (2003)

12. T. Messin, et al., Confinement effect in PC/MXD6 multilayer films: Impact of the microlayered structure on water and gas barrier properties, J. Membr. Sci. 525, 135 (2017)

13. A. Bironeau, J. Dirrenberger, C. Sollogoub, G. MiquelardGarnier, S. Roland, Evaluation of morphological representative sample sizes for nanolayered polymer blends, J. Microsc. 264(1), 48 (2016)

14. R. Bartolini, W. Hannan, D. Karlsons, M. Lurie, HOLOGRAPHY Embossed Hologram Motion Pictures for Television Playback, Appl. Opt. 9(10), 2283 (1970)

15. M.T. Gale, J. Kane, K. Knop, ZOD Images-Embossable Surface-Relief Structures for Color and Black-and-White Reproduction, J. Appl. Photogr. Eng. 4(2), 41 (1978)

16. E.W. Backer, et al., Production of separation-nozzle systems for uranium enrichment by a combination of X-ray lithography and galvanoplastics, Naturwissenschaften 69 (11), 520 (1982)

17. H. Vollmer, W. Ehrfeld, P. Hagmann, Fabrication of microstructures with extreme structural heights by vacuum reaction injection molding and electroforming, NASA STIRecon Tech. Rep. N 88, (1987)

18. M. Harmening, et al., Molding of three dimensional microstructures by the LIGA process, in: Proceedings IEEE Micro Electro Mechanical Systems, 1992, pp. 202-207

19. N. Okulova, P. Johansen, L. Christensen, R. Taboryski, Replication of micro-sized pillars in polypropylene using the extrusion coating process, Microelectron. Eng. 176, 54 (2017)

20. M. Röhrig, et al., Hot pulling and embossing of hierarchical nano- and micro-structures, J. Micromechanics Microengineering 23(10), 105014 (2013)

21. M. Cecchini, F. Signori, P. Pingue, S. Bronco, F. Ciardelli, F. Beltram, High-Resolution Poly(ethylene terephthalate) (PET) Hot Embossing at Low Temperature: Thermal, Mechanical, and Optical Analysis of Nanopatterned Films, Langmuir 24(21), 12581 (2008) 
22. J.V. Erps, et al., Hot Embossing of Microoptical Components Prototyped by Deep Proton Writing, IEEE Photonics Technol. Lett. 20(18), 1539 (2008)

23. M. Heckele, W.K. Schomburg, Review on micro molding of thermoplastic polymers, J. Micromechanics Microengineering 14(3), R1 (2004)

24. Aide-mémoire - Transformation des matières plastiques, 2017

25. Plastic Injection Molding Industry Expected to Grow by Almost 5\% Annually Through 2020, Magenta LLC, 2016

26. L'Injection des matières, Plastique Industries

27. Z. Tadmor, Molecular orientation in injection molding, J. Appl. Polym. Sci. 18(6), 1753 (1974)

28. R. Mendoza, Morphologies induites dans les pièces en polyolefine moulées par injection, $\mathrm{PhD}$ thesis, Paris, ENSAM, 2005

29. M. Vite, Relations entre microstructure, propriétés mécaniques et résistance à la rayure du polypropylène injecté, $\mathrm{PhD}$ thesis, université Savoie Mont Blanc, France, 2009

30. J. Giboz, T. Copponnex, P. Mélé, Microinjection molding of thermoplastic polymers: morphological comparison with conventional injection molding, J. Micromechanics Microengineering 19(2), 025023 (2009)

31. E.E. Ferg, L.L. Bolo, A correlation between the variable melt flow index and the molecular mass distribution of virgin and recycled polypropylene used in the manufacturing of battery cases, Polym. Test. 32(8), 1452 (2013)

32. H.-G. Elias, R. Bareiss, J.G. Watterson, Mittelwerte des Molekulargewichtes und anderer Eigenschaften, in Fortschritte der Hochpolymeren-Forschung, Springer, Berlin Heidelberg, 1973, pp. 111-204

33. M.M. Cross, Rheology of non-Newtonian fluids: A new flow equation for pseudoplastic systems, J. Colloid Sci. 20(5), 417 (1965)

34. P.J. Carreau, Rheological equations from molecular network theories, Trans. Soc. Rheol. 16(1), 99 (1972)

35. K. Yasuda, R.C. Armstrong, R.E. Cohen, Shear flow properties of concentrated solutions of linear and star branched polystyrenes, Rheol. Acta 20(2), 163 (1981)

36. C.A. Hieber, H.H. Chiang, Some correlations involving the shear viscosity of polystyrene melts, Rheol. Acta 28(4), 321 (1989)

37. T. Bremner, A. Rudin, D.G. Cook, Melt flow index values and molecular weight distributions of commercial thermoplastics, J. Appl. Polym. Sci. 41(7-8), 1617 (1990)

38. J. Vera, A.-C. Brulez, E. Contraires, M. Larochette, S. Valette, S. Benayoun, Influence of the polypropylene structure on the replication of nanostructures by injection molding, J. Micromechanics Microengineering 25(11), $115027(2015)$

39. R. Nakhoul, P. Laure, L. Silva, M. Vincent, A multiphase Eulerian approach for modelling the polymer injection into a textured mould, Int. J. Mater. Form., 1 (2016)

40. R.-D. Chien, W.-R. Jong, S.-C. Chen, Study on rheological behavior of polymer melt flowing through micro-channels considering the wall-slip effect, J. Micromechanics Microengineering 15(8), 1389 (2005)

41. A. Lamure, Mise en oeuvre des polymères. Disponible sur : http://www.inp-toulouse.fr/_resources/documents/ TICE/Mat\%25C3\%25A9riaux\%2520et\%2520polym\% 25C3\%25A8res/02Extrait_Mise_en_oeuvre_des_polyme res.pdf?download $=$ true [Consulté le $:-2017 / 1 \overline{8} / 04]$
42. M.S. Despa, K.W. Kelly, J.R. Collier, Injection molding of polymeric LIGA HARMs, Microsyst. Technol. 6(2), 60 (1999)

43. S.-C. Tseng, Y.-C. Chen, C.-L. Kuo, B.-Y. Shew, A study of integration of LIGA and M-EDM technology on the microinjection molding of ink-jet printers' nozzle plates, Microsyst. Technol. 12(1-2), 116 (2005)

44. S. Yuan, N.P. Hung, B.K.A. Ngoi, M.Y. Ali, Development of Microreplication Process-Micromolding, Mater. Manuf. Process. 18(5), 731 (2003)

45. M. Debowski, J. Zhao, A. Spowage, P. Glendenning, Development of techniques and methodologies for microand sub-micro evaluation of moulded polymer systems, Citeseer, (2003)

46. C.K. Huang, S.W. Chen, C.T. Yang, Accuracy and mechanical properties of multiparts produced in one mold in microinjection molding, Polym. Eng. Sci. 45(11), 1471 (2005)

47. H. Ito, H. Suzuki, K. Kazama, T. Kikutani, Polymer structure and properties in micro- and nanomolding process, Curr. Appl. Phys. 9(2), e19 (2009)

48. X. Lu, L.S. Khim, A statistical experimental study of the injection molding of optical lenses, J. Mater. Process. Technol. 113(1-3), 189 (2001)

49. Y.-C. Su, J. Shah, L. Lin, Implementation and analysis of polymeric microstructure replication by micro injection molding, J. Micromechanics Microengineering 14(3), 415 (2004)

50. A.W. McFarland, M.A. Poggi, L.A. Bottomley, J.S. Colton, Injection-moulded scanning force microscopy probes, Nanotechnology 16(8), 1249 (2005)

51. A.W. McFarland, M.A. Poggi, L.A. Bottomley, J.S. Colton, Injection moulding of high aspect ratio micron-scale thickness polymeric microcantilevers, Nanotechnology 15 (1), 1628 (2004)

52. H. Ito, Y. Yagisawa, T. Saito, T. Yasuhara, T. Kikutani, Y. Yamagiwa, Fundamental Study on Structure Development of Thin-Wall Injection Molded Products, Theor. Appl. Mech. Jpn. 54, 263 (2005)

53. N.B. Malhab, Moulage par microinjection des polymères semi-cristallins, $\mathrm{PhD}$ thesis, École nationale supérieure d'arts et métiers - ENSAM, 2012

54. G. Mougin, Principaux modes de dégradation des outillages en plasturgie, in Bulletin du cercle d'Études des Métaux, Pôle européen de plasturgie, Oyonnax, Vol. Tome XVII, 2015

55. R. Lévèque, Aciers à outils, évolution des nuances et de leurs traitements de surface, in Bulletin du cercle d'Études des Métaux, École des mines d'Albi, Vol. Tome XVIII, 2015

56. P. Jacquot, P. Foraison, Traitements appliqués aux outillages et moules d'injection plastique, in Bulletin du cercle d'Études des Métaux, Pôle européen de plasturgie, Oyonnax, Vol. Tome XVII, 2003

57. B. Saha, W.Q. Toh, E. Liu, S.B. Tor, D.E. Hardt, J. Lee, A review on the importance of surface coating of micro/nanomold in micro/nano-molding processes, J. Micromechanics Microengineering 26(1), 013002 (2016)

58. C.A. Griffiths, et al., A novel texturing of micro injection moulding tools by applying an amorphous hydrogenated carbon coating, Surf. Coat. Technol. 235, 1 (2013)

59. C. Donnet, A. Erdemir, Tribology of Diamond-like Carbon Films: Fundamentals and Applications, Springer Science \& Business Media, Berlin Heidelberg, 2007 
60. M. Chailly, Influence des traitements de surface de moule dans le procédé d'injection-moulage: application aux défauts d'aspect, $\mathrm{PhD}$ thesis, Villeurbanne, INSA, 2007

61. K. Reichelt, X. Jiang, The preparation of thin films by physical vapour deposition methods, Thin Solid Films 191 (1), 91 (1990)

62. S.M. Rossnagel, Thin film deposition with physical vapor deposition and related technologies, J. Vac. Sci. Technol. Vac. Surf. Films 21(5), S74 (2003)

63. J.E. Mahan, Physical Vapor Deposition of Thin Films, John Wiley \& Sons Inc, 2000, ISBN: 978-0-471-33001-1

64. T. Prieur, Sélection d'un précurseur pourl'élaboration de couchesatomiques de cuivre : application à l'intégration 3D - Recherche Google, PhD thesis, université de Grenoble, 2012

65. V.F. Neto, R. Vaz, M.S.A. Oliveira, J. Grácio, CVD diamond-coated steel inserts for thermoplastic mould toolsCharacterization and preliminary performance evaluation, J. Mater. Process. Technol. 209(2), 1085 (2009)

66. P. Loan, H. Prestavoine, Innovations BALINIT pour les outillages de frappe et d'injection, in Bulletin du cercle d'Études des Métaux, École des mines d'Albi, Vol. Tome XVIII, 2015

67. L. Cunha, et al., Performance of chromium nitride and titanium nitride coatings during plastic injection moulding, Surf. Coat. Technol. 153(2-3), 160 (2002)

68. L. Cunha, et al., Performance of chromium nitride based coatings under plastic processing conditions, Surf. Coat. Technol. 133-134, 61 (2000)

69. V. Miikkulainen, et al., Thin films of MoN, WN, and perfluorinated silane deposited from dimethylamido precursors as contamination resistant coatings on microinjection mold inserts, Surf. Coat. Technol. 202(21), 5103 (2008)

70. S.J. Bull, R.I. Davidson, E.H. Fisher, A.R. McCabe, A.M. Jones, A simulation test for the selection of coatings and surface treatments for plastics injection moulding machines, Surf. Coat. Technol. 130(2-3), 257 (2000)

71. S.-H. Yoon, et al., Effect of processing parameters, antistiction coatings, and polymer type when injection molding microfeatures, Polym. Eng. Sci. 50(2), 411 (2010)

72. M. Matschuk, N.B. Larsen, Injection molding of high aspect ratio sub-100 nm nanostructures, J. Micromechanics Microengineering 23(2), 025003 (2013)

73. C.A. Griffiths, S.S. Dimov, E.B. Brousseau, C. Chouquet, J. Gavillet, S. Bigot, Investigation of surface treatment effects in micro-injection-moulding, Int. J. Adv. Manuf. Technol. 47(1-4), 99 (2010)

74. T.C. Hobæk, M. Matschuk, J. Kafka, H.J. Pranov, N.B. Larsen, Hydrogen silsesquioxane mold coatings for improved replication of nanopatterns by injection molding, J. Micromechanics Microengineering 25(3), 035018 (2015)

75. M. Van Stappen, K. Vandierendonck, C. Mol, E. Beeckman, E. De Clercq, Practice vs. laboratory tests for plastic injection moulding, Surf. Coat. Technol. 142-144, 143 (2001)

76. J.-Y. Charmeau, M. Chailly, V. Gilbert, Y. Béreaux, Influence of mold surface coatings in injection molding. Application to the ejection stage, Int. J. Mater. Form. 1(1), 699 (2008)

77. P. Jones, Mould design guide, 2015
78. J.-Y. Chen, S.-J. Hwang, Design and fabrication of an adhesion force tester for the injection moulding process, Polym. Test. 32(1), 22 (2013)

79. X. Zhang, B. Sun, N. Zhao, Q. Li, J. Hou, W. Feng, Experimental study on the surface characteristics of Pdbased bulk metallic glass, Appl. Surf. Sci. 321, 420 (2014)

80. N. Bagcivan, K. Bobzin, T. Brögelmann, C. Kalscheuer, Development of ( $\mathrm{Cr}, \mathrm{Al}) \mathrm{ON}$ coatings using middle frequency magnetron sputtering and investigations on tribological behavior against polymers, Surf. Coat. Technol. 260, 347 (2014)

81. G. Zitzenbacher, Z. Huang, M. Längauer, C. Forsich, C. Holzer, Wetting behavior of polymer melts on coated and uncoated tool steel surfaces, J. Appl. Polym. Sci. 133(21), (2016)

82. C. Rytka, N. Opara, N.K. Andersen, P.M. Kristiansen, A. Neyer, On The Role of Wetting, Structure Width, and Flow Characteristics in Polymer Replication on Micro- and Nanoscale, Macromol. Mater. Eng. 301, 597 (2016)

83. J.M. Stormonth-Darling, R.H. Pedersen, C. How, N. Gadegaard, Injection moulding of ultra high aspect ratio nanostructures using coated polymer tooling, J. Micromechanics Microengineering 24 (7), 075019 (2014)

84. P. Roy, Microplasturgie, Techn. Ingenieur, AM3701 (2001)

85. J. Giboz, T. Copponnex, P. Mélé, Microinjection molding of thermoplastic polymers: a review, J. Micromechanics Microengineering 17(6), R96 (2007)

86. U.M. Attia, J.R. Alcock, A review of micro-powder injection moulding as a microfabrication technique, J. Micromechanics Microengineering 21(4), 043001 (2011)

87. Y. Xia, G.M. Whitesides, Soft Lithography, Angew. Chem. In t. Ed. 37(5), 550 (1998)

88. J.P. Rolland, E.C. Hagberg, G.M. Denison, K.R. Carter, J. M. De Simone, High-Resolution Soft Lithography: Enabling Materials for Nanotechnologies, Angew. Chem. 116(43), 5920 (2004)

89. A.K. Dubey, V. Yadava, Laser beam machining-A review, Int. J. Mach. Tools Manuf. 48(6), 609 (2008)

90. H.E. Jeong, M.K. Kwak, C.I. Park, K.Y. Suh, Wettability of nanoengineered dual-roughness surfaces fabricated by UVassisted capillary force lithography, J. Colloid Interface Sci. 339(1), 202 (2009)

91. S.-M. Lee, T.H. Kwon, Mass-producible replication of highly hydrophobic surfaces from plant leaves, Nanotechnology $\mathbf{1 7}$ (13), 3189 (2006)

92. R.A. Singh, E.-S. Yoon, H.J. Kim, J. Kim, H.E. Jeong, K.Y. Suh, Replication of surfaces of natural leaves for enhanced micro-scale tribological property, Mater. Sci. Eng. C 27(4), 875 (2007)

93. Y. Xue, Voie innovante pour la nano micro texturation de surfaces métalliques à base d'assemblage de nanoparticules d'Au : application superhydrophobe, PhD thesis, université Pierre et Marie Curie, Paris VI, 2014

94. Morphotonix, Industries. Disponible sur: http://www. morphotonix.com/industries/ [Consulté le: 2017/10/04]

95. J. Yang, Y. Zhao, X. Zhu, Transition between nonthermal and thermal ablation of metallic targets under the strike of high-fluence ultrashort laser pulses, Appl. Phys. Lett. 88(9), 094101 (2006)

96. B.N. Chichkov, C. Momma, S. Nolte, A. Von, A. Tünnermann, Femtosecond, picosecond and nanosecond laser ablation of solids, Appl. Phys. Mater. Sci. Process. 63 (2), 109 (1996) 
97. N.M. Bulgakova, I.M. Bourakov, Phase explosion under ultrashort pulsed laser ablation: Modeling with analysis of metastable state of melt, Appl. Surf. Sci. 197-198, 41 (2002)

98. A. Cavalleri, K. Sokolowski-Tinten, J. Bialkowski, M. Schreiner, D.L. Von, Femtosecond melting and ablation of semiconductors studied with time of flight mass spectroscopy, J. Appl. Phys. 85(6), 3301 (1999)

99. W.G. Roeterdink, L.B.F. Juurlink, O.P.H. Vaughan, D. Dura, M. Bonn, A.W. Kleyn, Coulomb explosion in femtosecond laser ablation of $\mathrm{Si}(111)$, Appl. Phys. Lett. 82(23), 4190 (2003)

100. R. Stoian, D. Ashkenasi, A. Rosenfeld, E.E.B. Campbell, Coulomb explosion in ultrashort pulsed laser ablation of Al2O3, Phys. Rev. B - Condens. Matter Mater. Phys. 62 (19), 13167 (2000)

101. J. Houzet, N. Faure, M. Larochette, A.-C. Brulez, S. Benayoun, C. Mauclair, Ultrafast laser spatial beam shaping based on Zernike polynomials for surface processing, Opt. Express 24(6), 6542 (2016)

102. É. Audouard, Lasers à impulsions ultrabrèves : applications, Tech. Ing.TIB452DUO, e6455 (2011)

103. N. Stutzmann, T.A. Tervoort, C.W.M. Bastiaansen, K. Feldman, P. Smith, Solid-State Replication of Relief Structures in Semicrystalline Polymers, Adv. Mater. 12 (8), 557 (2000)

104. T. Ibatan, M.S. Uddin, M.A.K. Chowdhury, Recent development on surface texturing in enhancing tribological performance of bearing sliders, Surf. Coat. Technol. 272, 102 (2015)

105. S. Hammouti, Micro-texturation de surface du PEEK par laser femtoseconde : étude locale de l'interaction laserpolymère et apport de la texturation de surface aux propriétés tribologiques d'un contact PEEK/PEEK, PhD thesis, École centrale de Lyon, Écully, 2015

106. P. Bizi Bandoki, Structuration multi-échelle d'alliages métalliques au moyen d'un laser Femtoseconde, Écully, École centrale de Lyon, 2012

107. J. Bonse, S. Baudach, J. Krüger, W. Kautek, M. Lenzner, Femtosecond laser ablation of silicon-modification thresholds and morphology, Appl. Phys. Mater. Sci. Process. 74 (1), 19 (2002)

108. T.-H. Her, R.J. Finlay, C. Wu, S. Deliwala, E. Mazur, Microstructuring of silicon with femtosecond laser pulses, Appl. Phys. Lett. 73(12), 1673 (1998)

109. P. Bizi-Bandoki, S. Benayoun, S. Valette, B. Beaugiraud, E. Audouard, Modifications of roughness and wettability properties of metals induced by femtosecond laser treatment, Appl. Surf. Sci. 257(12), 5213 (2011)

110. M. Birnbaum, Semiconductor Surface Damage Produced by Ruby Lasers, J. Appl. Phys. 36(11), 3688 (1965)

111. P.M. Fauchet, A.E. Siegman, Surface ripples on silicon and gallium arsenide under picosecond laser illumination, Appl. Phys. Lett. 40(9), 824 (1982)

112. L. Qi, F. Li, H. Lin, J. Hu, On the formation of regular subwavelength ripples by femtosecond laser pulses on silicon, Opt. - Int. J. Light Electron Opt. 126(24), 4905 (2015)

113. J.F. Young, J.S. Preston, D. Van, J.E. Sipe, Laser-induced periodic surface structure. II. Experiments on $\mathrm{Ge}, \mathrm{Si}, \mathrm{Al}$, and brass, Phys. Rev. B 27(2), 1155 (1983)

114. J.E. Sipe, J.F. Young, J.S. Preston, H.M. van Driel, Laserinduced periodic surface structure. I. Theory, Phys. Rev. B 27(2), 1141 (1983)
115. J. Reif, et al., On large area LIPSS coverage by multiple pulses, Appl. Surf. Sci. 336, 249 (2015)

116. K.M.B. Jansen, D.J. Van Dijk, M.H. Husselman, Effect of processing conditions on shrinkage in injection molding, Polym. Eng. Sci. 38(5), 838 (1998)

117. K.M.B. Jansen, R. Pantani, G. Titomanlio, As-molded shrinkage measurements on polystyrene injection molded products, Polym. Eng. Sci. 38(2), 254 (1998)

118. H.-Y. Lin, W.-B. Young, Analysis of the filling capability to the microstructures in micro-injection molding, Appl. Math. Model. 33(9), 3746 (2009)

119. N. Zhang, J.S. Chu, C.J. Byrne, D.J. Browne, M.D. Gilchrist, Replication of micro/nano-scale features by micro injection molding with a bulk metallic glass mold insert, J. Micromechanics Microengineering 22(6), 065019 (2012)

120. K. Mönkkönen, et al., Replication of sub-micron features using amorphous thermoplastics, Polym. Eng. Sci. 42(7), $1600(2002)$

121. V. Kalima, et al., Transparent thermoplastics: Replication of diffractive optical elements using micro-injection molding, Opt. Mater. 30(2), 285 (2007)

122. J. Zhao, R.H. Mayes, G. Chen, H. Xie, P.S. Chan, Effects of process parameters on the micro molding process, Polym. Eng. Sci. 43(9), 1542 (2003)

123. B. Sha, S. Dimov, C. Griffiths, M.S. Packianather, Investigation of micro-injection moulding: Factors affecting the replication quality, J. Mater. Process. Technol. 183(23), 284 (2007)

124. I. Ariño, U. Kleist, G.G. Barros, P.-A. Johansson, M. Rigdahl, Surface texture characterization of injectionmolded pigmented plastics, Polym. Eng. Sci. 44(9), 1615 (2004)

125. E. Huovinen, L. Takkunen, M. Suvanto, T.A. Pakkanen, Fabrication and quantitative roughness analysis of hierarchical multiscale polymer surface structures, J. Micromechanics Microengineering 24(5), 055017 (2014)

126. C. Rytka, P.M. Kristiansen, A. Neyer, Iso- and variothermal injection compression moulding of polymer micro- and nanostructures for optical and medical applications, J. Micromechanics Microengineering 25(6), 065008 (2015)

127. V. Bellantone, R. Surace, G. Trotta, I. Fassi, Replication capability of micro injection moulding process for polymeric parts manufacturing, Int. J. Adv. Manuf. Technol. 67(5-8), 1407 (2013)

128. M. Matschuk, H. Bruus, N.B. Larsen, Nanostructures for allpolymer microfluidic systems, Microelectron. Eng. 87(5-8), 1379 (2010)

129. J. Chu, M.R. Kamal, S. Derdouri, A. Hrymak, Characterization of the microinjection molding process, Polym. Eng. Sci. 50(6), 1214 (2010)

130. V. Miikkulainen, T. Rasilainen, E. Puukilainen, M. Suvanto, T.A. Pakkanen, Atomic Layer Deposition as Pore Diameter Adjustment Tool for Nanoporous Aluminum Oxide Injection Molding Masks, Langmuir 24(9), 4473 (2008)

131. J.M. Dealy, Rheometers for molten plastics: a practical guide to testing and property measurement, Van Nostrand Reinhold Company, Springer-Verlag, Berlin Heidelberg, 1982 ISBN-13: 978-0442218744

132. R. Ballman, T. Shusman, Easy way to calculate injection molding set-up time, Mod. Plast. 126, 130 (1959)

133. G.R. Berger, D.P. Gruber, W. Friesenbichler, C. Teichert, M. Burgsteiner, Replication of Stochastic and Geometric 
Micro Structures - Aspects of Visual Appearance, Int. Polym. Process. 26(3), 313 (2011)

134. D. Yao, S.-C. Chen, B.H. Kim, Rapid thermal cycling of injection molds: An overview on technical approaches and applications, Adv. Polym. Technol. 27(4), 233 (2008)
135. M.J. Liou, N.P. Suh, Reducing residual stresses in molded parts, Polym. Eng. Sci. 29(7), 441 (1989)

136. G. Lucchetta, E. Ferraris, G. Tristo, D. Reynaerts, Influence of mould thermal properties on the replication of micro parts via injection moulding, Procedia CIRP 2, 113 (2012)

Citation de l'article : Julie Vera, Anne-Catherine Brulez, Elise Contraires, Mathieu Larochette, Stéphane Valette, Stéphane Benayoun, Synthèse bibliographique : micro-texturation et microinjection de thermoplastiques, Matériaux \& Techniques 105, 303 $(2017)$ 